\title{
Two-Dimensional Particle-In-Cell Simulation of Magnetic Sails
}

\section{AUTHOR(S):}

Ashida, Yasumasa; Funaki, Ikkoh; Yamakawa, Hiroshi; Usui, Hideyuki; Kajimura, Yoshihiro; Kojima, Hirotsugu

\section{CITATION:}

Ashida, Yasumasa ...[et al]. Two-Dimensional Particle-In-Cell Simulation of Magnetic Sails. Journal of Propulsion and Power 2014, 30(1): 233-245

\section{ISSUE DATE:}

2014-01

URL:

http://hdl.handle.net/2433/182205

\section{RIGHT:}

(C) AIAA; この論文は出版社版でありません。引用の際には出版社版を ご確認ご利用ください。; This is not the published version. Please cite only the published version. 


\title{
Two-Dimensional Particle-In-Cell Simulation of Magnetic Sails
}

\author{
Yasumasa Ashida ${ }^{1}$ \\ Kyoto University, Kyoto, 611-0011, Japan \\ Ikkoh Funaki ${ }^{2}$ \\ Japan Aerospace Exploration Agency, Kanagawa, 252-5210, Japan \\ Hiroshi Yamakawa ${ }^{3}$ \\ Kyoto University, Kyoto, 611-0011, Japan \\ Hideyuki Usui ${ }^{4}$ \\ Kobe University, Hyogo, 657-8501, Japan \\ Yoshihiro Kajimura ${ }^{5}$ \\ Akashi National College of Technology, Hyogo, 674-8501, Japan \\ and \\ Hirotsugu Kojima ${ }^{6}$ \\ Kyoto University, Kyoto, 611-0011, Japan
}

Magnetic sail is spacecraft propulsion that produces an artificial magnetosphere to block solar wind particles, and thus impart momentum to accelerate a spacecraft. In the present study, we conducted two-dimensional particle-in-cell simulations on small-scale magnetospheres to investigate thrust characteristics of magnetic sail and its derivative, Magneto Plasma Sail (MPS), in which the magnetosphere is inflated by an additional plasma injection. As a result, we found that the electron Larmor motion and the charge separation become significant on such a small-scale magnetosphere and the thrust of magnetic sail is affected by the cross-sectional size of charge-separated magnetosphere. We also revealed that the plasma injection on the condition that the kinetic energy of plasma is smaller than the local magnetic field energy $\left(\beta \sim 10^{-3}\right)$ can significantly inflate the magnetosphere by

\footnotetext{
${ }^{1}$ Graduate student, Division of Creative Research and Development of Humanosphere, Research Institute for Sustainable Humanosphere, Gokasyo, Uji.

${ }^{2}$ Associate Professor, Institute of Space and Astronautical Science, 3-1-1 Yoshinodai, Sagamihara, Senior Member AIAA.

${ }^{3}$ Professor, Division of Creative Research and Development of Humanosphere, Research Institute for Sustainable Humanosphere, Gokasyo, Senior Member AIAA.

${ }^{4}$ Professor, Graduate School of System Informatics, 1-1 Rokkodai, Nada, Kobe.

${ }^{5}$ Lecturer, Department of Electric and Computer Engineering, 679-3 Nisioka, Uozumi-cho, Akashi, Member AIAA.

${ }^{6}$ Associate Professor, Division of Creative Research and Development of Humanosphere, Research Institute for Sustainable Humanosphere, Gokasyo, Uji.
} 
inducing diamagnetic current in the same direction as the onboard coil current. As a result, the MPS thrust is increased effectively by an additional plasma injection: the MPS thrust (15 $\mu \mathrm{N} / \mathrm{m})$ becomes up to 7.5 times larger than the original thrust of the magnetic sail (2.0 $\mu \mathrm{N} / \mathrm{m})$. In addition, we found that the thrust gain of MPS defined as "MPS thrust /

(Magnetic sail thrust + plasma injection thrust)" becomes up to 2.2.

\section{Nomenclature}

$\mathbf{B}=$ magnetic flux density vector, $\mathrm{T}$

$B_{M P}=$ magnetic flux density at magnetopause, $\mathrm{T}$

$B_{i n j}=$ magnetic flux density at plasma injection source, $\mathrm{T}$

$C_{d}=$ thrust coefficient

$c \quad=\quad$ light speed, $\mathrm{m} / \mathrm{s}$

$\mathrm{d} t \quad=\quad$ time step, $\mathrm{s}$

$\mathrm{d} x=$ grid spacing, $\mathrm{m}$

$\mathbf{E}=$ electric field vector, $\mathrm{V} / \mathrm{m}$

$E_{M P}=$ electric field at magnetopause, $\mathrm{V} / \mathrm{m}$

$e \quad=\quad$ elementary charge, $\mathrm{C}$

$F_{\text {mag }}=$ thrust by magnetic sail, $\mathrm{N} / \mathrm{m}$

$F_{M P S}=\quad$ thrust by MPS, N/m

$F_{\text {jet }}=$ equivalent thrust by plasma injection from MPS spacecraft, N/m

$F_{\text {plasma }}=\quad$ force acting on diamagnetic plasma current

$I_{\text {coil }}=$ coil current, $\mathrm{A}$

$I_{\text {plasma }}=$ plasma current by plasma injection, $\mathrm{A}$

$I_{s p} \quad=\quad$ specific impulse, $\mathrm{s}$

$\mathbf{J}=$ plasma current density vector, $\mathrm{A} / \mathrm{m}^{2}$

$J_{M P}=$ magnetopause current density, $\mathrm{A} / \mathrm{m}^{2}$

$k_{B}=$ Boltzmann constant, $\mathrm{J} / \mathrm{K}$

$L=$ representative length of magnetosphere obtained by theory using MHD approximation, $\mathrm{m}$ 


$$
\begin{aligned}
& M \quad=\quad \text { magnetic moment of coil, } \mathrm{Wbm} \\
& m_{i} \quad=\quad \text { mass of ion, } \mathrm{kg} \\
& m_{e} \quad=\quad \text { mass of electron, } \mathrm{kg} \\
& N_{S W}=\text { number density of the solar wind plasma, } \mathrm{m}^{-3} \\
& N_{i n j}=\text { number density of the injected plasma, } \mathrm{m}^{-3} \\
& n_{i}=\text { number density of ion, } \mathrm{m}^{-3} \\
& n_{e}=\text { number density of electron, } \mathrm{m}^{-3} \\
& Q=\text { flow rate of plasma injection, } \mathrm{m}^{2} / \mathrm{s} \\
& q_{i}=\text { charge of ion, }+e \mathrm{C} \\
& q_{e}=\text { charge of electron, }-e \mathrm{C} \\
& R_{\text {coil }}=\text { coil distance from the coil center, } \mathrm{m} \\
& R_{\text {plasma }}=\quad \text { plasma current distance from the coil center, } \mathrm{m} \\
& r_{i L}=\text { Larmor radius of ion at magnetopause, } \mathrm{m} \\
& r_{e L}=\text { Larmor radius of electron at magnetopause, } \mathrm{m} \\
& T_{S W}=\text { solar wind plasma temperature, } \mathrm{eV} \\
& T_{i n j, i}=\quad \text { temperature of injected ion, } \mathrm{eV} \\
& T_{i n j, e}=\text { temperature of injected electron, } \mathrm{eV} \\
& \mathbf{u}_{i n j}=\text { fluid velocity of injected plasma, } \mathrm{m} / \mathrm{s} \\
& V_{S W}=\text { velocity of solar wind plasma, } \mathrm{m} / \mathrm{s} \\
& V_{i n j, i}=\quad \text { velocity of the injected ion, } \mathrm{m} / \mathrm{s} \\
& V_{i n j, e}=\quad \text { velocity of the injected electron, } \mathrm{m} / \mathrm{s} \\
& \mathbf{v}_{i}=\text { velocity vector of ion particle, } \mathrm{m} / \mathrm{s} \\
& \mathbf{v}_{e}=\text { velocity vector of electron particle, } \mathrm{m} / \mathrm{s} \\
& \alpha=\text { attack angle, degree } \\
& \beta_{i n j}=\text { beta of injected plasma at injection source } \\
& \lambda_{D}=\text { Debye length, } \mathrm{m} \\
& \mu_{0} \quad=\quad \text { permeability of vacuum, N/A }{ }^{2}
\end{aligned}
$$




\section{Introduction}

W AGNETIC sail is a spacecraft propulsion system that aims at future interplanetary flight for deep space explorations. Zubrin and Andrews first provided the concept of magnetic sail in 1991 [1]. As shown in Fig. 1, they conceptually designed a spacecraft with a large loop of a superconductive coil. The onboard superconductive coil produces an artificial magnetic cavity (magnetosphere) to reflect solar wind particles approaching the coil. Due to this interaction, the solar wind flow loses its momentum, and a corresponding repulsive force exerts on the coil to accelerate the magnetic sail spacecraft in the anti-sunward direction without consuming propellant. Due to an unrealistically large coil structure ( $\sim$ several kilo meters in diameter) necessary for a magnetic sail, the magnetic sail did not gain much interest. In 2000, the concept of magnetic sail attained renewed interest when the idea to make a large magnetosphere and a corresponding large thrust by employing a compact coil ( $\sim$ several meters in diameter) with a plasma jet was proposed by Winglee et al. [2] instead of deploying a large-scale coil by Zubrin. Winglee's concept is illustrated in Fig. 2.

Early investigations [2] suggested that the propulsion system that is called as Mini-Magneto Plasma Propulsion (M2P2) or Magneto Plasma Sail (MPS) was a promising space propulsion system, which might drastically shorten the trip time of deep space missions. These investigations were based on the assumptions that a significant thrust force proportional to the area of a magnetosphere could be produced with a compact coil that can be equipped onboard a spacecraft. To understand M2P2/MPS performance, many studies were conducted using MagnetoHydoroDynamics (MHD) and Hybrid Particle-in-Cell (PIC) techniques. Table 1 represents the summary of previous studies [3-9]. From these simulations, it was revealed that the thrust by M2P2/MPS, $F_{M P S}$, is larger than that of magnetic sail, $F_{m a g}$, (i.e., $F_{M P S} / F_{m a g}>1$ ), supporting Winglee's concept. The thrust by M2P2/MPS were, however, limited as $F_{M P S} / F_{m a g}<10$. A detailed explanation for the limited thrust was proposed by Nishida et al. [4], who performed ideal-MHD simulation to obtain the maximum $F_{M P S} / F_{m a g}$ of about 2 for a low kinetic beta plasma jet; in contrast, for high kinetic beta plasma jets, $F_{M P S} / F_{m a g}$ became approximately zero even though the magnetospheric size was intensively inflated. A much better thrust performance was obtained in the case of ion inertial scale magnetospheres, in which $F_{M P S} / F_{m a g}$ up to 8 was obtained by Hybrid-PIC simulation with a low kinetic beta plasma jet [8]. These simulations showed that the idea of M2P2/MPS was rather promising in ion inertial scale magnetospheres, but $F_{M P S} / F_{\text {mag }}$ was not as large as expected in the early investigations. In fact, to make the M2P2/MPS system effective, $F_{M P S}$ should be larger than a net thrust force, $F_{m a g}+F_{j e t}$, in which the contribution from 
plasma jet $\left(F_{j e t}\right)$ is also incorporated. Summarizing the results obtained by the MHD and Hybrid-PIC simulations, we found that the effective thrust gain $F_{M P S} /\left(F_{m a g}+F_{j e t}\right)$ never exceeded unity (i.e., $\left.F_{M P S} /\left(F_{m a g}+F_{j e t}\right)=<1\right)$, and this means that no effective configuration of M2P2/MPS was found in the previous studies. It is also noted that these simulations did not include Interplanetary Magnetic Field (IMF), which may significantly affect the above obtained thrust gain. In the Earth's magnetosphere, IMF plays a very important role since the magnetic reconnection distorts the structure of the magnetosphere and transfers the momentum of plasma through the magneto tail. Only Nishida et al. [5] reflected the influence of IMF in thrust by performing 2D ideal-MHD simulation. However, it is difficult to reproduce the phenomena of a magnetic reconnection correctly by the ideal-MHD simulation including artificial viscosity and numerical diffusion.

To determine the maximum thrust gain available by M2P2/MPS, one needs to properly evaluate the thrust characteristics of M2P2/MPS, and for that purpose, fully kinetic simulation including all the related physical phenomena on M2P2/MPS seems the best solution. Full-PIC simulation adopted in this study treats both ions and electrons as particles, and it can solve the plasma flow around the magnetosphere including IMF self-consistently. In addition, the all spacecraft model proposed by Winglee et al., Cattell et al. [10] and Japan Aerospace Exploration Agency (JAXA) (Fig. 3) can be assumed directly, without any assumptions, only in Full-PIC simulation. However, in spite of the necessity for fully kinetic simulation, it has not yet been performed because of the huge computational requirement [11].

The objective of the present study is to reveal the thrust characteristics of magnetic sail and M2P2/MPS with small magnetospheres from several $100 \mathrm{~m}$ to several $1000 \mathrm{~m}$, where the electron kinetics should be considered. Two-dimensional Full-PIC method was employed since the fundamental physics did not change between twodimension or three-dimension, the two-dimensional analysis was adopted in order to reduce the computational resource. In the following, we first evaluate the finite thrust generation by the electron inertial scale magnetosphere including the electron kinetics and IMF. Next, we aim at further improvement in net thrust gain with the various plasma injection parameters. In order to achieve the above objectives, we performed Full-PIC simulations with the realistic mass ratio $\left(m_{i} / m_{e} \sim 1836\right)$ for hydrogen plasmas. 


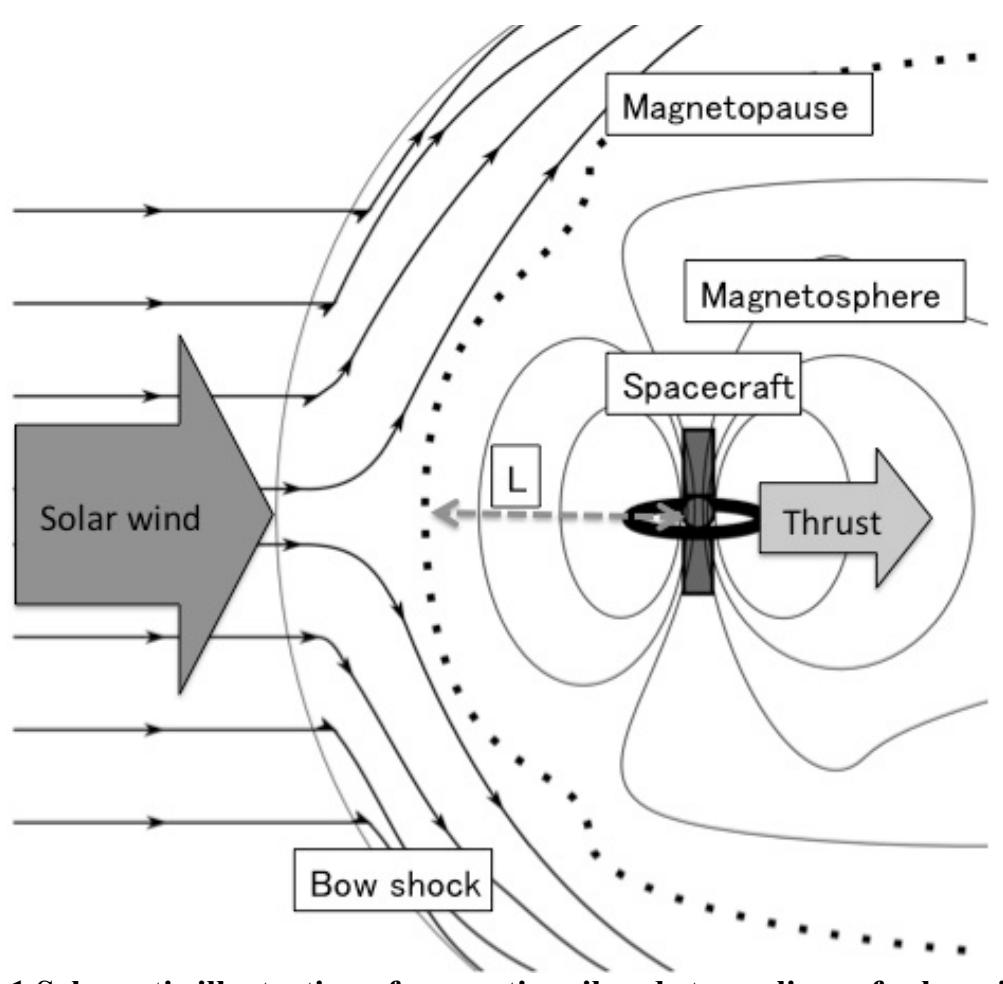

Fig. 1 Schematic illustration of magnetic sail and streamlines of solar wind.
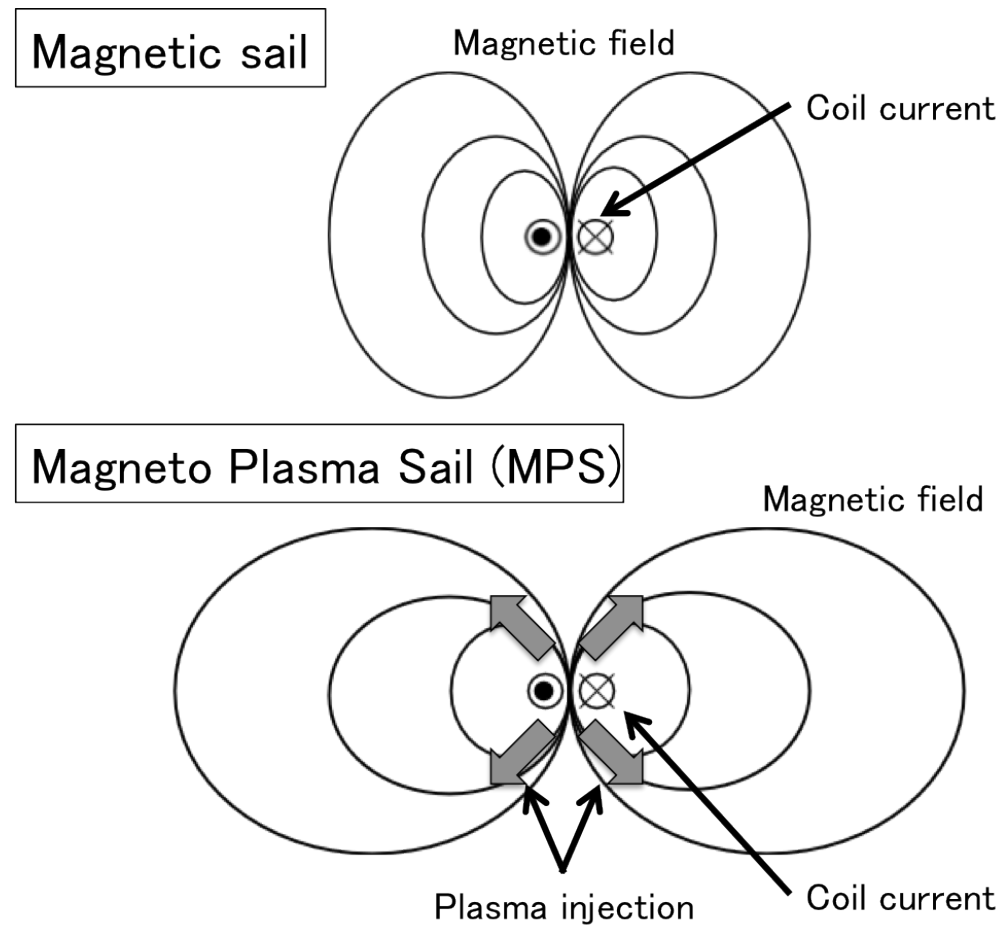

Fig. 2 Schematic illustration of magnetic sail and MPS. By a plasma injection, the dipole magnetic field of the magnetic sail is expanded. 


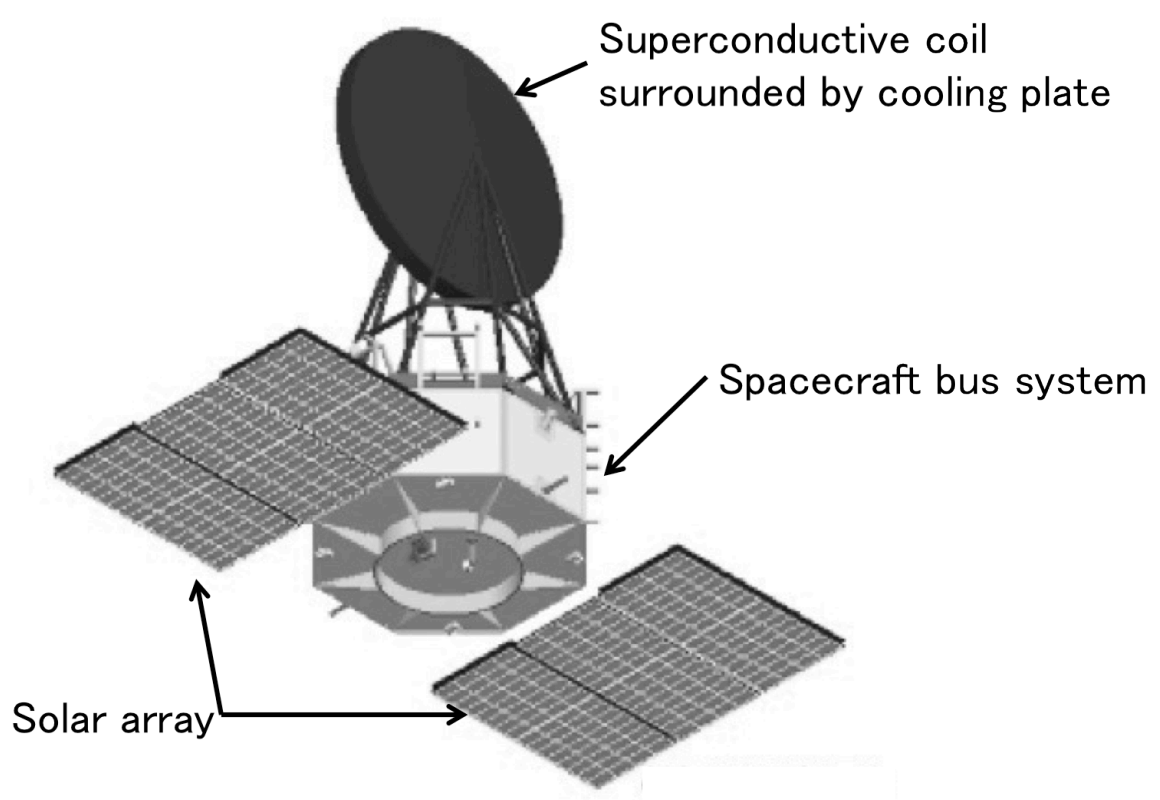

Fig. 3 M2P2/MPS spacecraft model proposed by JAXA. A $200 \mathrm{~kg}$ superconductive coil and $100 \mathrm{mN}$-class thrust generation is required.

Table 1 Previous studies about magnetic sail and M2P2/MPS by numerical simulation

\begin{tabular}{|c|c|c|c|c|c|c|c|}
\hline & Target & Dimension & $\begin{array}{l}\text { Simulation } \\
\text { method }\end{array}$ & Ion kinetics & $\begin{array}{l}\text { Electron } \\
\text { kinetics }\end{array}$ & IMF & $\begin{array}{l}\text { Thrust-mass } \\
\text { ratio and } I_{s p}\end{array}$ \\
\hline $\begin{array}{l}\text { Nishida et } \\
\text { al., 2006 [3] }\end{array}$ & Magnetic sail & $2 \mathrm{D}$ & MHD & N/A & N/A & N/A & N/A \\
\hline $\begin{array}{l}\text { Nishida et } \\
\text { al., } 2007 \text { [4] }\end{array}$ & M2P2/MPS & $3 \mathrm{D}$ & MHD & N/A & N/A & N/A & N/A \\
\hline $\begin{array}{l}\text { Nishida et } \\
\text { al., 2012 [5] }\end{array}$ & Magnetic sail & $2 \mathrm{D}$ & MHD & N/A & N/A & Considered & N/A \\
\hline $\begin{array}{l}\text { Khazanov et } \\
\text { al., } 2005 \text { [6] }\end{array}$ & M2P2/MPS & $3 \mathrm{D}$ & $\begin{array}{l}\text { MHD and } \\
\text { Hybrid-PIC }\end{array}$ & Considered & N/A & N/A & N/A \\
\hline $\begin{array}{l}\text { Winske et al., } \\
2005 \text { [7] }\end{array}$ & $\begin{array}{l}\text { Plasma } \\
\text { expansion }\end{array}$ & $3 \mathrm{D}$ & Hybrid-PIC & Considered & N/A & N/A & N/A \\
\hline $\begin{array}{l}\text { Kajimura et } \\
\text { al., } 2010[8]\end{array}$ & M2P2/MPS & $3 \mathrm{D}$ & Hybrid-PIC & Considered & N/A & N/A & N/A \\
\hline $\begin{array}{l}\text { Moritaka et } \\
\text { al., 2010 [9] }\end{array}$ & $\begin{array}{l}\text { Plasma } \\
\text { expansion }\end{array}$ & $2 \mathrm{D}$ & Full-PIC & Considered & $\begin{array}{l}\text { Considered } \\
\left(m_{\mathrm{i}} / m_{\mathrm{e}}=25\right)\end{array}$ & N/A & N/A \\
\hline Present study & $\begin{array}{l}\text { Magnetic sail } \\
\text { and M2P2/MPS }\end{array}$ & $2 \mathrm{D}$ & Full-PIC & Considered & $\begin{array}{l}\text { Considered } \\
\left(m_{\mathrm{i}} / m_{\mathrm{e}}=1836\right)\end{array}$ & Considered & Considered \\
\hline
\end{tabular}




\section{Numerical Model}

\section{A. Assumption}

The solar wind mainly consists of ions (protons) and electrons. Typical parameters of the solar wind at an Earth orbit are listed in Table 2. In the case of a magnetic sail in the interplanetary space, an ion mean free path is approximately $1.5 \times 10^{8} \mathrm{~km}$. It is much longer than the typical length of a magnetosphere we consider, $L=100 \mathrm{~m} \sim 10$ $\mathrm{km}$. Hence, direct collisions between particles can be neglected in the magnetic sail simulations. Here, the magnetospheric size $L$ shown in Fig. 1 is derived from the pressure equilibrium at the magnetopause (the boundary of the magnetosphere, where the magnetopause current is induced by the solar wind plasma). Based on MHD approximation, the solar wind dynamic pressure balances the magnetic pressure of the $2 \mathrm{D}$ dipole magnetic field generated by a magnetic sail spacecraft as Eq. (1). By solving Eq. (1) about $L$, the magnetospheric size is obtained as Eq. (2) with the MHD approximation.

$$
\begin{gathered}
\frac{1}{2} N_{S W} m_{i} V_{S W}^{2}=\frac{B^{2}}{2 \mu_{0}}=\frac{1}{2 \mu_{0}}\left(\frac{\mu_{0} R_{\text {coil }} I_{\text {coil }}}{2 \pi L^{2}}\right)^{2} \\
L=\left(\frac{\mu_{0} R_{\text {coil }}^{2} I_{\text {coil }}^{2}}{4 \pi^{2} N_{S W} m_{i} V_{S W}^{2}}\right)^{\frac{1}{4}}
\end{gathered}
$$

The interaction between solar wind plasma particles and the magnetosphere with size $L$ is characterized by Larmor motion in the magnetic field. When the typical parameters of the solar wind, $N_{S W}=5 \times 10^{6} \mathrm{~m}^{-3}, V_{S W}=5 \times 10^{5} \mathrm{~m} / \mathrm{s}$ are assumed, the magnetic flux density at the magnetopause $B_{M P}$ is estimated approximately as $50 \mathrm{nT}$ by the MHD approximation. The ion Larmor radius and the electron Larmor radius at the magnetopause are calculated as $r_{i L} \sim 100$ $\mathrm{km}$ and $r_{e L} \sim 50 \mathrm{~m}$, respectively. If $L$ and the ion Larmor radius $r_{i L}$ satisfy the condition of $L \gg>r_{i L}$, the finite Larmor effect can be neglected and the plasma flow can be treated as a single fluid on the MHD scale. On the contrary, if $L$ and $r_{i L}$ are comparable and $L \gg>r_{e L}$ at the magnetopause, the ion's finite Larmor radius effect should be considered and electrons can be assumed as a fluid on the ion inertial scale. When $L$ is smaller than $r_{i L}$, both ion's and electron's finite Larmor radius effect should be considered. We call this scale length of the magnetosphere $\left(L<r_{i L}\right.$ and $\left.L>r_{e L}\right)$ as the electron inertial scale. Full-PIC simulation is performed to reveal the thrust characteristics of magnetic sail and MPS on the electron inertial scale. 
Table 2 Typical parameters of the solar wind

\begin{tabular}{cccc}
\hline \hline Solar wind velocity $V_{S W}[\mathrm{~m} / \mathrm{s}]$ & Solar wind density $N_{S W}\left[\mathrm{~m}^{-3}\right]$ & Plasma temperature $T_{S W}[\mathrm{eV}]$ & Interplanetary magnetic field [T] \\
\hline $5 \times 10^{5}$ & $5 \times 10^{6}$ & 10 & 0 (ignored) \\
\hline \hline
\end{tabular}

\section{B. Basic equations}

Full-PIC simulation treats both ions and electrons as particles in order to consider the finite Larmor radius effects. In addition, the charge separation and the effects of the electric field at the magnetopause are considered. Full-PIC simulation solves the equation of motion, Eq. (3), and traces the precise motion of each ion and electron using the Buneman-Boris method [12]. From the particle trajectories, a density distribution and a current distribution are obtained according to the PIC weighting method. Maxwell equations, Eqs. (4) and (5), are solved by using the Finite-difference time-domain (FDTD) method to obtain a self-consistent electromagnetic field. The electric field is corrected electrostatically by Poisson's equation in order to conserve the energy.

The interaction between the solar wind and an artificial dipole magnetic field is simulated in two-dimensional space ( $x-z$ plane). The computational domain used by two-dimensional Full-PIC simulations is shown in Fig. 4a. The computational domain has an area of $15 \mathrm{~km} \times 15 \mathrm{~km}$ involving $512 \times 512$ grids in the typical case. The grid spacing $\mathrm{d} x$ should be chosen not much larger than the Debye length $\left(\mathrm{d} x / \lambda_{D}<3\right)$ and typically 16 super particles are involved in a grid. The original two-dimensional dipole magnetic field is calculated using the opposite current $\pm I_{\text {coil }}$. The coil currents are defined as the corresponding current density $\left(I_{\text {coil }} / 2 \mathrm{~d} x^{2}\right)$ at grid center of two source grids $\left( \pm R_{\text {coil }}\right)$. The attack angle $\alpha$ is defined on the basis of the solar wind (z-axis) direction as shown in Fig. $4 \mathrm{~b}$. In this study, cases of $\alpha=0{ }^{\circ}$ (the magnetic moment of the coil is parallel to the solar wind direction) and $\alpha=90^{\circ}$ (the magnetic moment is perpendicular to the solar wind direction) are simulated. The absorbing boundary condition is set for the electromagnetic field in all boundaries. The solar wind particles flow into the computational domain from the inflow boundary with the typical solar wind velocity and the thermal distribution. The outgoing particles from the computational domain are eliminated from the calculation. In addition, as mentioned in section IV, artificial plasma is injected from the injection source grid near the coil for the MPS simulations as shown in Fig. 4a. 
The thrust generated by magnetic sail and MPS is calculated using two different methods. In the first method, the electromagnetic force between the induced current by a plasma flow $\mathbf{J}$ and the coil current onboard spacecraft $I_{\text {coil }}$ is calculated, and the electromagnetic force is designated as $\mathbf{F}_{\text {mag. }}$. The second method uses a change in the solar wind momentum passing over a control volume to derive a thrust, $\mathbf{F}_{\text {flow }}$. In the first method, the magnetic field near a coil is calculated based on the Biot-Savart law as in Eq. (6). In the second method, the thrust is calculated by an arbitrary surface integral of the momentum as in Eq. (7). Note that $\mathbf{u}_{i} \mathbf{u}_{i}, \mathbf{B B}$, and $\mathbf{I}$ are tensors. The terms in Eq. (7) represent the momentum exchange by ion motions, the magnetic pressure and the Maxwell stresses, respectively. The thrust calculated by the second method is only correct when the calculation is steady state.

$$
\begin{gathered}
m_{s} \frac{d \mathbf{v}_{s}}{d t}=q_{s}\left(\mathbf{E}+\mathbf{v}_{s} \times \mathbf{B}\right) \quad s=\text { ion, electron } \\
\frac{\partial \mathbf{B}}{\partial t}=-\nabla \times \mathbf{E} \\
\frac{1}{c^{2}} \frac{\partial \mathbf{E}}{\partial t}=\nabla \times \mathbf{B}-\mu_{0} \mathbf{J} \\
\mathbf{F}_{\text {mag }}=\left(\sum \sum \frac{\mu_{0} \mathbf{J} S \times \mathbf{r}}{2 \pi r^{2}}\right) \times \mathbf{I}_{\text {coil }} \\
\mathbf{F}_{\text {flow }}=-\oint\left(m_{i} n_{i} \mathbf{u}_{i} \mathbf{u}_{i}+\frac{\mathbf{B} \cdot \mathbf{B}}{2 \mu_{0}} \mathbf{I}-\frac{\mathbf{B B}}{\mu_{0}}\right) \cdot \mathbf{n} d s
\end{gathered}
$$




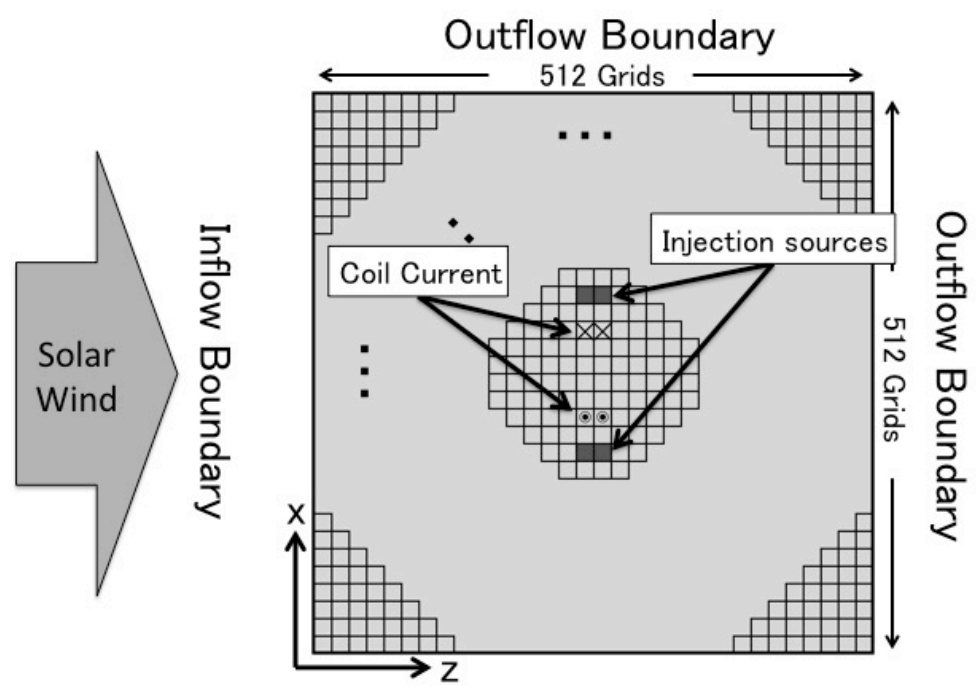

a)

Outflow Boundary

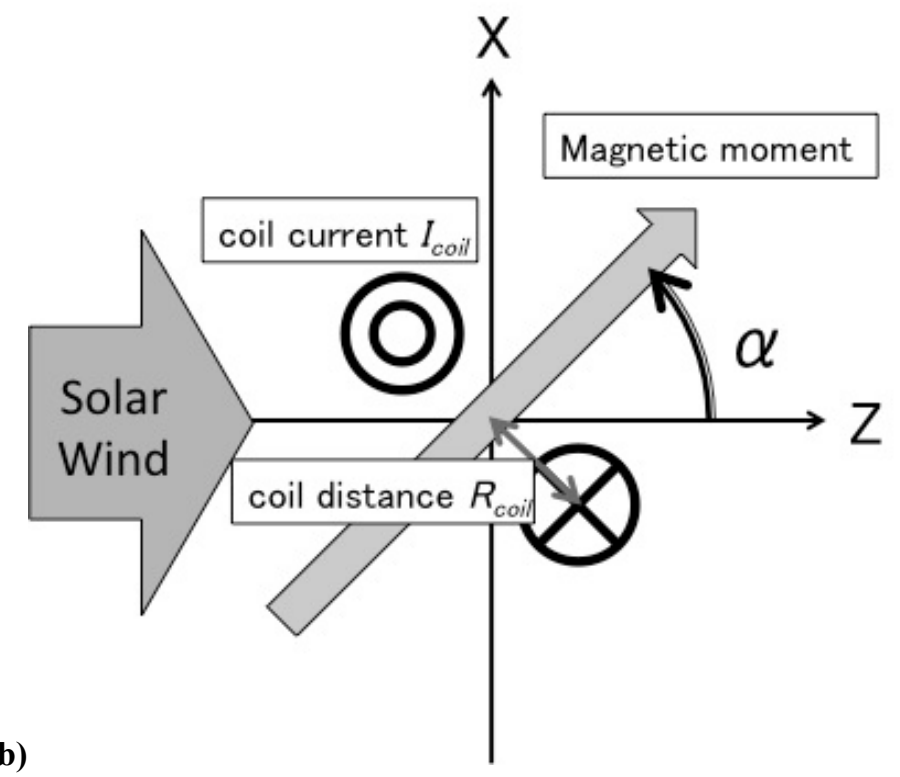

Fig. 4 a) Computational domain used for the two-dimensional Full-PIC simulations and b) definition of the attack angle $\alpha$. 


\section{Thrust characteristics of magnetic sails}

\section{A. Typical two-dimensional magnetosphere of a magnetic sail}

First, to demonstrate how the momentum is transferred from the solar wind to the spacecraft with the small-scale magnetosphere affected by the electron kinetics, we performed Full-PIC simulation without a plasma injection. Two-dimensional magnetic sail with the parameters listed in Table 3 was simulated in order to clarify the effects of electron's finite Larmor radius and charge separation on the flow and magnetic field of the small-scale magnetic sail. We consider only an artificial dipole magnetic field without taking into account IMF. From Eq. (2), theoretical magnetospheric size $L$ is calculated as $L=2200 \mathrm{~m}$ under the conditions in Table 3, and hence $L<r_{i L}(100 \mathrm{~km})$ and $L>r_{e L}(50 \mathrm{~m})$ are satisfied. This case corresponds to a magnetosphere on the electron inertial scale. Figure 5a shows the spatial distribution of ion density in steady state when the magnetic dipole moment is perpendicular to the solar wind direction $\left(\alpha=90^{\circ}\right)$. In Fig. 5a, a low density region so-called 'wake region' is formed around the origin, $(x$, $z)=(0,0)$, in spite of loose coupling between the ions and the magnetosphere $\left(r_{i L}>L\right)$. Similar but slightly larger wake region also appears in Fig. 5b, which shows the electron density distribution.

Figures $6 \mathrm{a}$ and $6 \mathrm{~b}$ show the schematic illustration of the plasma density distribution and simulation results of the plasma density, the electric field and the current density along $z$-axis $(x=0 \mathrm{~m})$, respectively. Here, the plasma density represents $n_{i}-n_{e}$. At the magnetopause $(-1000 \mathrm{~m}<x<-900 \mathrm{~m})$, an electron-rich region is formed since electrons cannot penetrate into the magnetic field because of their small Larmor radius $r_{e L}$. On the contrary, ion is abundant in the interior of the magnetosphere $(x>-900 \mathrm{~m})$. By charge separation between ions and electrons at the magnetopause, the outward electric field $E_{M P}$ appears as illustrated in Fig. 6a. The trajectories of ions close to the magnetopause are bent by $E_{M P}$. Consequently, the ion density around spacecraft becomes low nevertheless ions are not magnetized. On the contrary, the magnetized electrons make $\mathbf{E} \times \mathbf{B}$ drift motions at the magnetopause, thus the magnetopause current $J_{M P}$ that flows in the counter direction of $I_{\text {coil }}$ is induced. The Lorentz force between $J_{M P}$ and the onboard coil current $I_{c o i l}$ works as thrust by magnetic sail as illustrated in Fig. 6 a.

Dimensions of the wake are determined from the distance between the origin and the magnetopause current peak. From Fig. 6b, the magnetospheric size in $z$-direction is calculated as $900 \mathrm{~m}$ in the present parameters. Following the same procedure, dimensions of the wake are determined as $900 \mathrm{~m}$ (stagnation length) $\times 1100 \mathrm{~m}$ (cross-sectional length), and these are quite small compared with the theoretical value $L=2200 \mathrm{~m}$. By using Full-PIC simulation, the 
electron's finite Larmor effect and charge separated structure of magnetosphere are revealed for the first time, which are impossible to be analyzed ether by MHD simulation or Hybrid-PIC simulation.

Finally, the thrust of magnetic sail is obtained as a force per unit length. To check self-consistency of the simulation, thrust is evaluated by two methods: the Lorentz force between $\mathbf{J}$ and $I_{\text {coil }}$ and the momentum change of solar wind particles. The thrust is calculated as $2.0 \times 10^{-6} \pm 0.1 \times 10^{-6} \mathrm{~N} / \mathrm{m}$. To check the dependency of the thrust on particle numbers and grid size, simulations with different conditions are also performed: particle number (4 particles / cell, 16 particles / cell, 64 particles / cell), grid number $(256 \times 256,512 \times 512)$ and grid spacing $\mathrm{d} x(15 \mathrm{~m}, 30 \mathrm{~m}, 60$ m). As a result, it was found that the thrust level is overestimated by approximately $20 \%$ when the computational domain is smaller than the typical case $(512 \times 512, \mathrm{~d} x=30 \mathrm{~m})$ due to the influence of the boundary. When the grid spacing is $\mathrm{d} x=60 \mathrm{~m}$, the thrust is calculated as $2.2 \times 10^{-6} \pm 0.2 \times 10^{-6} \mathrm{~N} / \mathrm{m}$. The error bar becomes larger by rough grid spacing. On the contrary, no clear difference appears to the thrust by the diffrences of particle number. As the necessary and sufficient calculation condition, 16 partilces / cell is used in the remainder of this study.
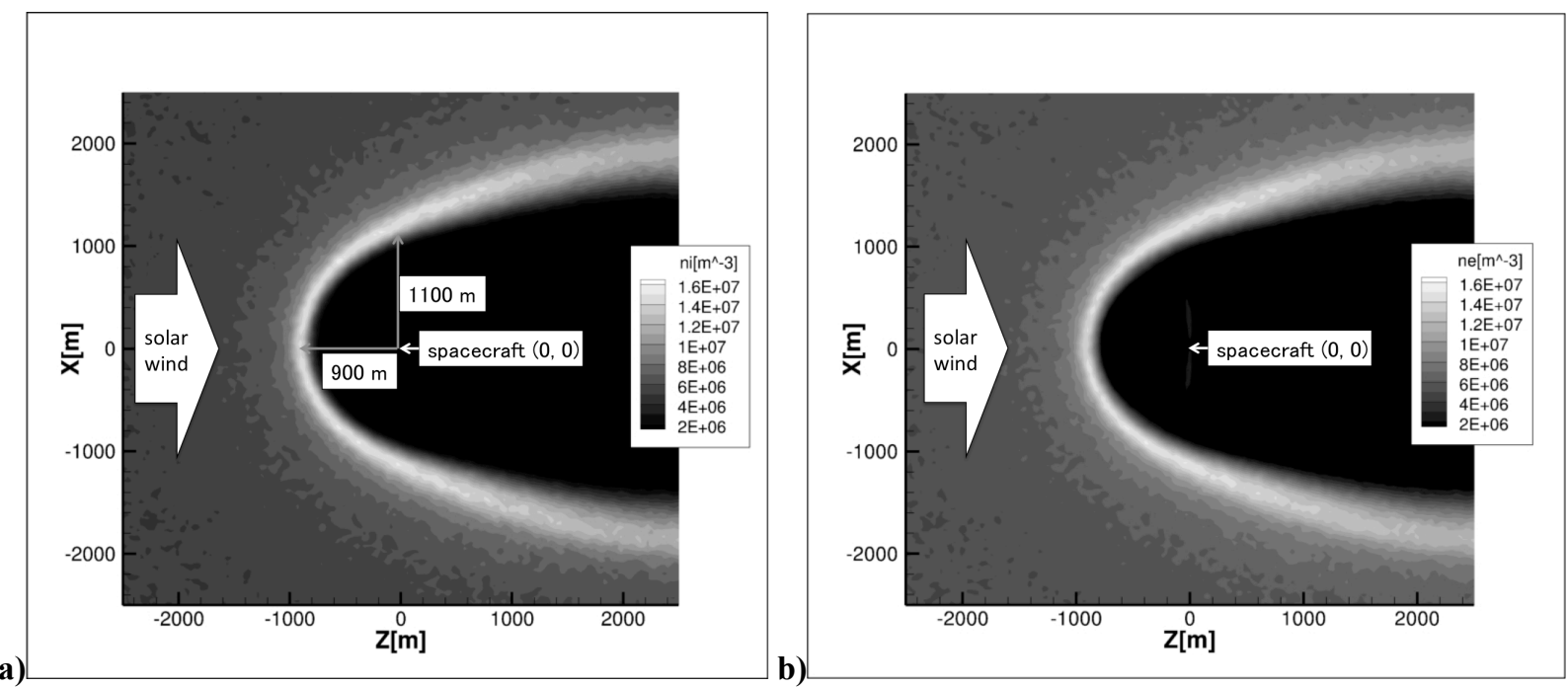

Fig. 5 Density distribution of ion and electron (magnetic sail, $L=2200 \mathrm{~m}, \alpha=90^{\circ}$ ): a) ion density and b) electron density. 
a)
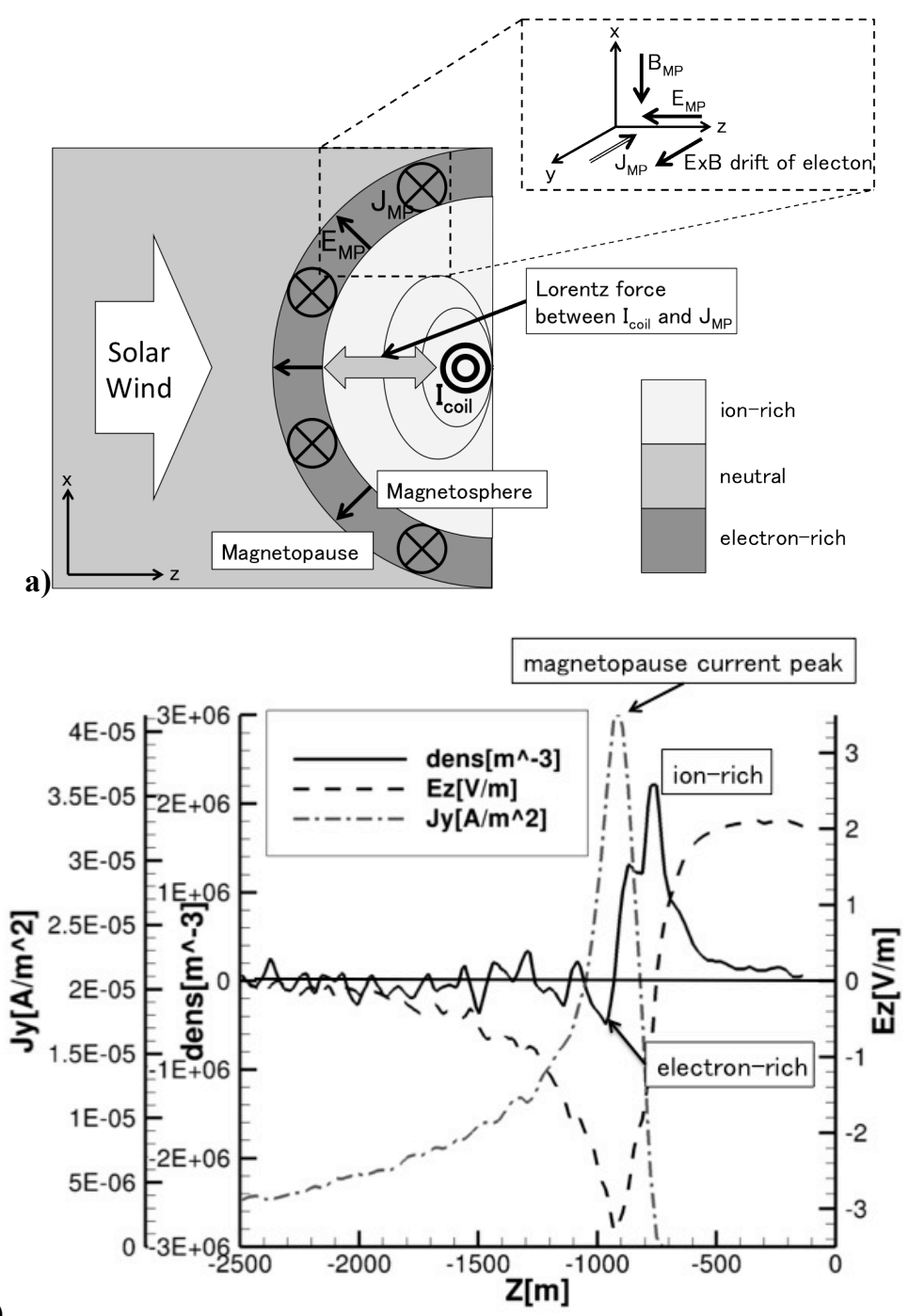

b)

Fig. 6 a) Schematic illustration of magnetopause and b) one-dimensional distributions of plasma density, electric field and current density along $z$-axis $(x=0 \mathrm{~m})$ in steady state by simulation (magnetic sail, $L=2200 \mathrm{~m}, \alpha=90^{\circ}$ ).

Table 3 Magnetic sail design parameters

\begin{tabular}{cc}
\hline \hline Magnetic sail parameters & \\
\hline Coil distance $R_{\text {coil }}$ & $75 \mathrm{~m}$ \\
Coil current $\times$ Turn $I_{\text {coil }}$ & $4.0 \times 10^{3} \mathrm{~A}$ Turn \\
Magnetospheric size $L$ & $2.2 \times 10^{3} \mathrm{~m}$ \\
Simulation parameters & \\
\hline Grid, $\mathrm{d} x$ & $30 \mathrm{~m}$ \\
Time, $\mathrm{d} t$ & $4.0 \times 10^{-8} \mathrm{~s}$ \\
Grid number & $512 \times 512$ \\
\hline \hline
\end{tabular}


In addition to the simulations neglecting the effects of IMF, we performed the simulation with IMF as shown in Fig. 7. Although the magnetosphere in MHD scale has the discontinuity of the magnetic field around the magnetopause current peak, Fig. 7a represents that the magnetic discontinuity is not observed in electron inertial scale magnetosphere even if IMF is neglected since the plasma flow can induce the only weaker magnetopause current than the coil current. This is because a larger Larmor radius of ion and electron than the magnetospheric size causes the loose coupling between the plasma flow and the magnetic field. In Fig. 7b, The magnetic flux density and the direction of IMF are set as $7 \mathrm{nT}$ and $45 \mathrm{deg}$., that is, $\left(B_{x}, B_{z}\right)=(5 \mathrm{nT}, 5 \mathrm{nT})$ and the similar magnetosphere to Fig. $7 \mathrm{a}$ is formed as shown in Fig. $7 \mathrm{~b}$. The thrust per unit length is also calculated as $1.9 \times 10^{-6} \pm 0.1 \times 10^{-6} \mathrm{~N} / \mathrm{m}$. That is, it was revealed that the thrust of magnetic sail is hardly influenced by IMF in electron inertial scale as well as MHD scale [5]. Hence, in the remainder of this study, we consider only an artificial dipole magnetic field without taking into account IMF for simplicity.
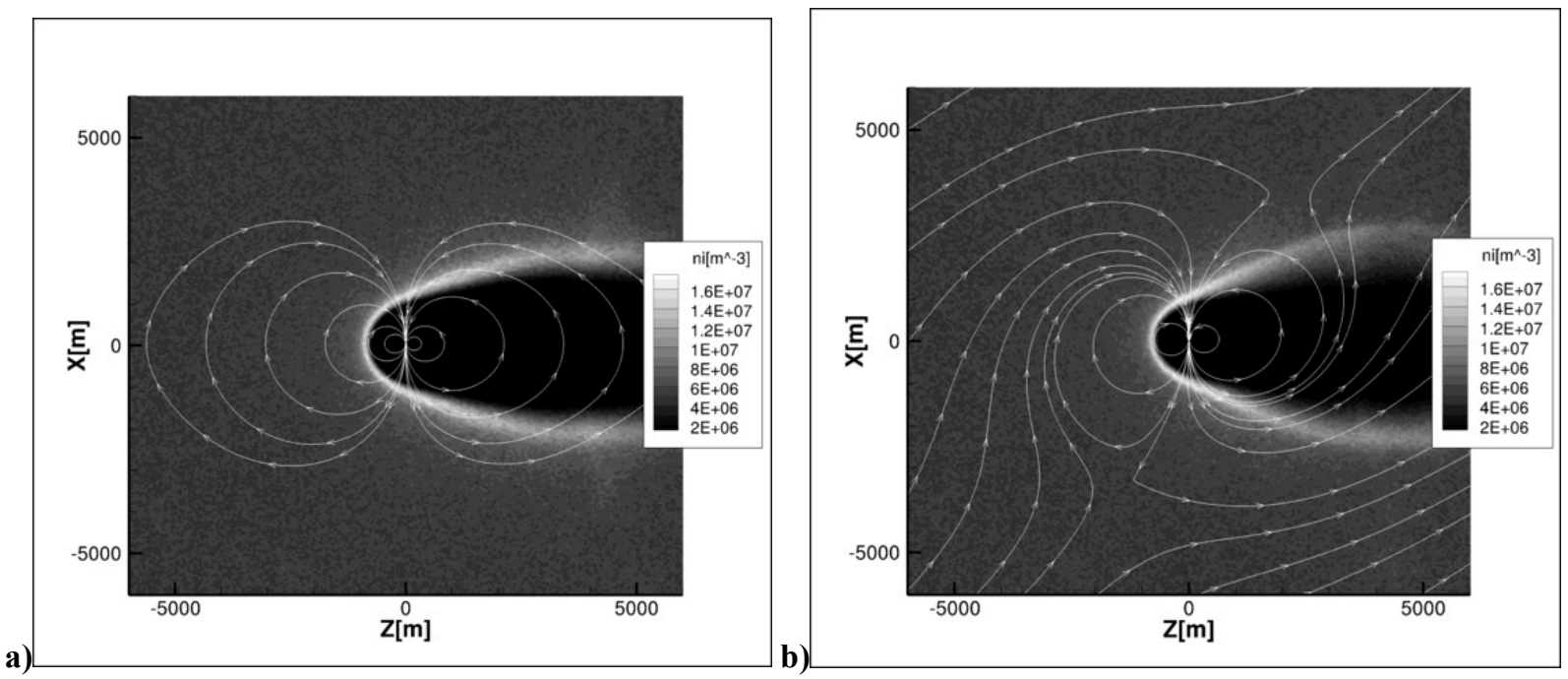

Fig. 7 Ion density distribution and magnetic field line of electron scale magnetosphere without IMF(a) and with $\operatorname{IMF}(b)$ (magnetic sail, $L=2200 \mathrm{~m}, \alpha=90^{\circ}$ ). The magnetic moment is perpendicular to the solar wind direction(z-axis). The absolute value and the direction of IMF are set as $7 \mathrm{nT}$ and 45 deg., respectively. 


\section{B. Thrust characteristics of magnetic sail for various magnetospheric sizes}

The thrust characteristics are analyzed for various magnetospheric sizes. The theoretical magnetospheric size $L$ was selected from $380 \mathrm{~m}$ to $4300 \mathrm{~m}$ depending on the coil current $I_{\text {coil }}=13$ to $1.6 \times 10^{4} \mathrm{~A}$ (electron inertial scale $\left.L<<r_{i L}\right)$. The thrust characteristics of larger magnetospheric size equivalent to MHD scale $\left(L>>r_{i L}\right)$ and ion inertial scale $\left(L \sim r_{i L}\right)$ have been already analyzed by MHD simulation $[3,5,13]$, Hybrid-PIC simulation $[14,15,16]$ and Flux-Tube simulation [17] and the thrust characteristics of small magnetospheric size remains to be analyzed. The attack angle is $\alpha=0^{\circ}$ or $\alpha=90^{\circ}$. Parameters about magnetic sails and the simulation are listed in Table 4 and Table 5, respectively. Results are shown in Fig. 8, in which the solid line shows the results of magnetic sail in the parallel case $\left(\alpha=0^{\circ}\right)$ and the dotted line shows the results of magnetic sail in the perpendicular case $\left(\alpha=90^{\circ}\right)$. Two important features are found from Fig. 8a: 1) rapid increase in thrust as the magnetospheric size becomes larger; and 2) thrust in the parallel case is larger than the thrust in the perpendicular case for the same magnetospheric size. The item 2) indicates that the magnetosphere in parallel case is larger than the magnetosphere in the perpendicular case, and it is supported by Fig. 9 which shows the ion density distribution of the parallel case and the perpendicular case for $L=2200 \mathrm{~m}$. As one can see, the cross-sectional lengths of the magnetosphere along $x$-axis $(z=0 \mathrm{~m})$ are obtained as $1100 \mathrm{~m}$ in the perpendicular case (Fig. 9a) and $1300 \mathrm{~m}$ in the parallel case (Fig. 9b). More particles hence interact with the parallel magnetosphere than the perpendicular magnetosphere due to the size effect, but seeing the upstream region in Fig. 9b, it is also found that the mirror magnetic field in the parallel case reflects the particles that approach the coil onboard the spacecraft. In addition to the size effect, the mirror magnet will contribute to produce a larger thrust in the case of parallel magnetosphere.

Non-dimensional force, or the drag coefficient, $C_{d}$, is defined by Eq. (8). $C_{d}$ is plotted in Fig. 8b, and Fig. 8c shows the ratio of the cross-sectional length obtained by Full-PIC simulation to the theoretical magnetospheric size $L$. In larger magnetosphere where the dipole approximation is valid $\left(L>>R_{\text {coil }}\right)$, the ratio increases monotonically and asymptotically approaches to unity as $L$ increases. On the contrary, in the case of small magnetosphere $(L<1000 \mathrm{~m}$ or cross-sectional length $<400 \mathrm{~m})$, the ratio does not monotonically increase since the finite coil size $\left(R_{\text {coil }}=75 \mathrm{~m}\right)$ cannot be negligible and the magnetic flux density equivalent to the magnetopause $\left(B_{M P} \sim 50 \mathrm{nT}\right)$ of coil magnetic field is extended radially compared with the magnetic flux density of an ideal dipole magnetic field. By the extended magnetic field, the cross-sectional length of the smaller magnetosphere becomes larger than that formed by an ideal dipole magnetic field. When the dipole approximation is valid, $C_{d}$ in the perpendicular case becomes larger at a 
constant rate in proportion to the increase in the ratio (cross-sectional length $/ L$ ) as represented by the dotted line in Fig. $8 \mathrm{~b}$ since the interactions between the solar wind and the magnetic field are enhanced according to the crosssectional length rather than the theoretical $L$. On the contrary, $C_{d}$ becomes larger rapidly from $L=770 \mathrm{~m}$ to $2200 \mathrm{~m}$ in the parallel case represented by the solid line in Fig. $8 \mathrm{~b}$ and the change rate of $C_{d}$ from $2200 \mathrm{~m}$ to $4300 \mathrm{~m}$ becomes smaller. It is expected that the mirror magnetic field cause this feature of $C_{d}$ in the parallel case as the biggest difference of the plasma flow from in the perpendicular case. The influence of the mirror magnetic field is considered as follows. The magnetic flux density at the coil center $\left(\mu_{0} I_{\text {coil }} / \pi R_{\text {coil }}\right)$ is proportional to $I_{\text {coil }}$ and the magnetospheric size $L$ represented by Eq. (2) is proportional to square root of $I_{\text {coil }}$. The mirror ratio is approximately assumed as $B_{M P} /\left(\mu_{0} I_{\text {coil }} / \pi R_{\text {coil }}\right)$ using the typical magnetic flux density at magnetopause $B_{M P}(\sim 50 \mathrm{nT}$, constant $)$ and the magnetic flux density at the coil center. The mirror ratio hence becomes smaller in inverse proportional to the square of the theoretical $L$. That is, more particles that approach the coil in the parallel case are reflected, as the theoretical $L$ becomes larger. These particles are expected to be mainly contributed to the thrust increase in the parallel case from $L=770 \mathrm{~m}$ to $2200 \mathrm{~m}$ since both ions and electrons slip away the magnetopause because of the large finite Larmor radius $\left(r_{i L}>>L\right.$ and $\left.r_{e L} \sim L\right)$ as same as particles in the perpendicular case without the mirror magnetic field. In the larger magnetosphere from $L=2200 \mathrm{~m}$ to $4300 \mathrm{~m}\left(r_{i L}>L\right.$ and $\left.r_{e L}<L\right)$, particles interact with the magnetic field perpendicular to the particle velocity at magnetopause rather than the mirror magnetic field. As a result, $C_{d}$ from $L=2200 \mathrm{~m}$ to $4300 \mathrm{~m}$ in the parallel case becomes larger at a constant rate in proportion to the increase in the ratio of the cross-sectional length of the magnetosphere and the theoretical $L$ as same as in the perpendicular case.

Thrust of magnetic sail, thus, depends on the cross-sectional length of the charge-separated magnetosphere affected by the finite Larmor effect and the mirror magnetic field. MPS is hence expected to be able to increase thrust by expanding the cross-sectional length of the magnetosphere. In addition, by reflecting the solar wind particles by the mirror magnetic field, the thrust increase beyond the increase in the cross-sectional length of the magnetosphere is expected in the parallel case. 
Table 4 Simulation parameters for various magnetospheric sizes

\begin{tabular}{cc}
\hline \hline Simulation parameters & \\
\hline Grid $\mathrm{d} x$ & $30 \mathrm{~m}$ \\
Time $\mathrm{d} t$ & $4.0 \times 10^{-8} \mathrm{~s}$ \\
Grid number & $512 \times 512$ \\
\hline \hline
\end{tabular}

Table 5 Magnetic sail design parameters for various magnetospheric sizes

\begin{tabular}{|c|c|c|c|c|c|c|c|c|}
\hline \multicolumn{9}{|l|}{ Magnetic sail parameters } \\
\hline Coil distance $R_{\text {coil }}[\mathrm{m}]$ & 75 & 75 & 75 & 75 & 75 & 75 & 75 & 75 \\
\hline Coil current $\times$ Turn $I_{\text {coil }}[$ A Turn $]$ & $1.3 \times 10^{1}$ & $2.5 \times 10^{2}$ & $5.0 \times 10^{2}$ & $1.0 \times 10^{2}$ & $2 \times 10^{3}$ & $4.0 \times 10^{3}$ & $8.0 \times 10^{3}$ & $1.6 \times 10^{4}$ \\
\hline Magnetospheric size $L[\mathrm{~m}]$ & $3.8 \times 10^{2}$ & $5.4 \times 10^{2}$ & $7.7 \times 10^{2}$ & $1.1 \times 10^{3}$ & $1.5 \times 10^{3}$ & $2.2 \times 10^{3}$ & $3.1 \times 10^{3}$ & $4.3 \times 10^{3}$ \\
\hline
\end{tabular}




$$
C_{d}=\frac{F_{m a g}}{\frac{1}{2} N_{S W} m_{i} V_{S W}^{2}(2 L)}
$$

a)
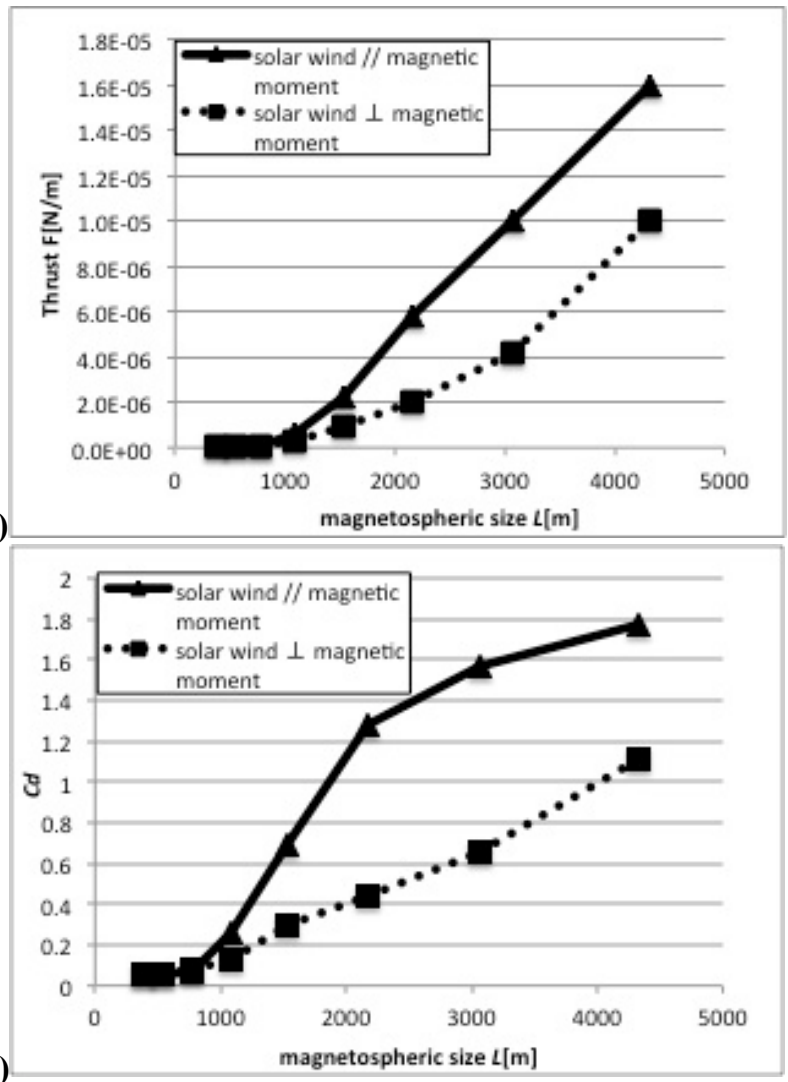

b)

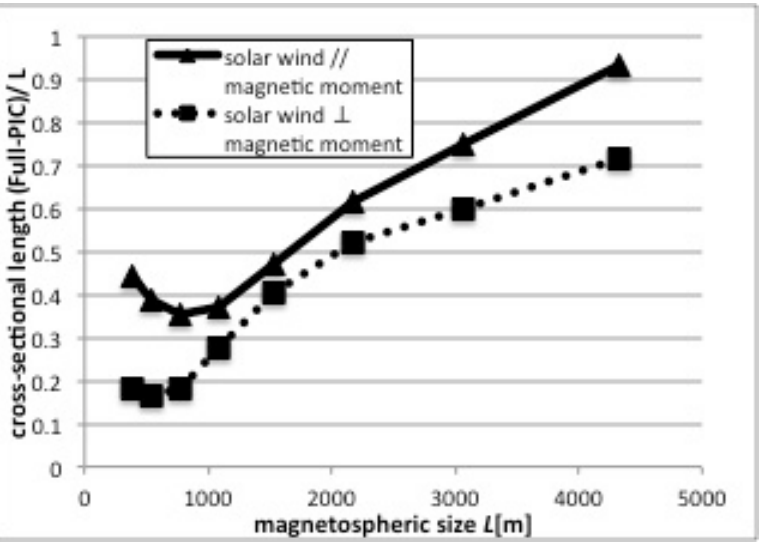

Fig. 8 Thrust characteristics of magnetic sails about magnetospheric size $L$ : a) thrust level $F_{m a g}$, b) drag coefficient $C_{d}$ and c) ratio of the cross-sectional length obtained by the simulations and the theoretical value $L$. The magnetospheric size varied from $380 \mathrm{~m}$ to $4300 \mathrm{~m}$ by changing the coil current. 

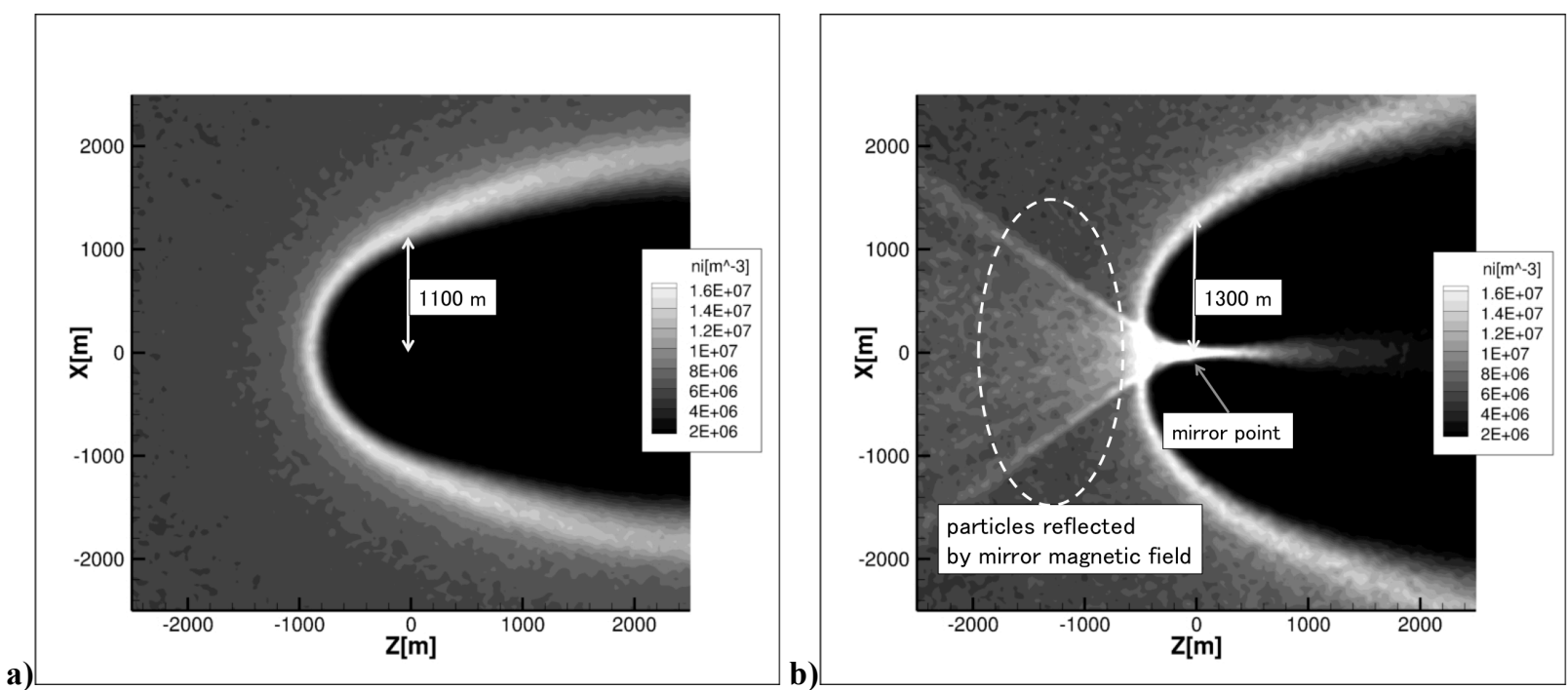

Fig. 9 Ion density distribution (magnetic sail, $L=2200 \mathrm{~m}$ ): a) perpendicular case $\left(\alpha=90{ }^{\circ}\right)$ and b) parallel case $\left(\alpha=0{ }^{\circ}\right)$. The particles reflected by the mirror magnetic field in the parallel case form the density distribution like a cone. 


\section{Thrust increase by a plasma injection: MPS}

\section{A. Typical simulation results of MPS on the electron inertial scale}

Several simulations of MPS have been previously performed. Hybrid-PIC simulations with the ion kinetic effects were performed to examine the expansion of the magnetosphere without the solar wind flow under different initial condition $[7,18,19]$. As a result, these studies revealed that the magnetic field can be expanded depending on ion Larmor radius and Alfven speed of the injected plasma even if the ion kinetc effect is considered. By using 2D FullPIC simulation including the electron kinetics, Moritaka et al. [9] also demonstrated the magnetic expanison without the solar wind plasma by assuming the artificial mass ratio $\left(m_{i} / m_{e} \sim 25\right)$. However, the thrust of MPS was never evaluated by Full-PIC simulation despite of the demonstrative analysis by MHD and Hybrid-PIC simulation [4, 6, 8]. In addition, the interaction between the solar wind and the inflated magnetic field is still unknown when the electron kinetics is taken into the consideration (see also Table 1).

According to the previous studies, to obtain a large magnetosphere and the increase in thrust, MPS spacecraft can use two methods. One is to expand a magnetosphere by a high-density and high-velocity plasma jet from a magnetic sail spacecraft, and the other is to initiate a ring-current inside a magnetosphere by releasing slow velocity plasma [20]. In the first method which is originally proposed by Winglee et al, when plasma jet is totally magnetized (i.e., the MHD approximation is valid), the plasma flow and the magnetic field move together according to Eq. (9), whose condition is usually called as 'frozen-in'. Here, as for the injection plasma, the characteristic length of magnetic field is considered as $B / \nabla B$ at an injection source. The plasma under the frozen-in condition, hence, satisfies the condition of $r_{i L}, r_{e L}<<B / \nabla B$. Under the frozen-in condition, the magnetic field is conveyed along with a plasma flow to a far region from spacecraft, resulting in a larger magnetosphere. The frozen-in concept is, however, not suitable for magnetic field inflation if the injected plasma, particularly ion, is not magnetized (i.e., finite Larmor effect appears, $r_{i L} \sim B / \nabla B$ or $\left.r_{i L}>B / \nabla B\right)$, since the plasma flow will escape from the magnetosphere. Instead, the second method for magnetic field inflation relies on a diamagnetic current induced by particle motion. Figure 10 shows the schematic illustration of a magnetized electron motion in a two-dimensional dipole magnetic field. In this case, $\nabla B$ drift motion of electrons induces a current flow in the same direction as $I_{\text {coil }}$ and therefore the induced current will increase the magnetic moment, hence thrust will be increased. When a lot of electrons are added, the collective motion of electrons increases the current that flows in the same direction as $I_{c o i l}$, hence thrust by MPS is 
expected to further increase. Total current induced by the drift motion and the collective motion is called as the diamagnetic current $I_{\text {plasma }}$.

$$
\frac{\partial \mathbf{B}}{\partial t}=\nabla \times\left(\mathbf{u}_{i n j} \times \mathbf{B}\right)
$$

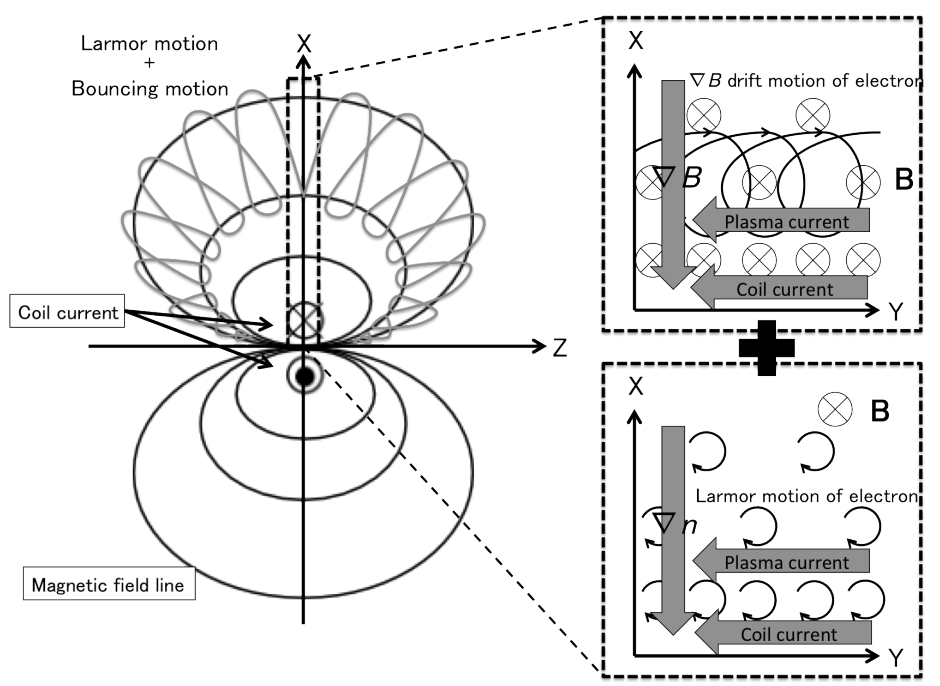

Fig. 10 Schematic illustration of a magnetized electron in the two-dimensional dipole magnetic field. An electron moves along the magnetic field line while making Larmor motion and bounces at the mirror point. In addition, electrons induce the diamagnetic current that flows in the same direction as the coil current.

The Full-PIC simulation of MPS is, then, performed in two-dimension. The solar wind parameters for MPS simulation are the same as the parameters listed in Table 2. The theoretical magnetospheric size obtained by Eq. (2) is $L=2200 \mathrm{~m}$, and the value of $L$ is fixed throughout MPS simulations. In addition to the solar wind parameters, magnetic sail design parameters (for electromagnet) and injection plasma parameters for magnetic field inflation are selected as shown in Tables 6 and 7, respectively. When releasing plasma from MPS spacecraft, as shown in Fig. 4a, four plasma injection source cells located at $x= \pm 150$ are defined around the coil current, and super particles are added to the injection source cells at a rate of once every 100 steps in the simulation. The plasma is injected toward the magnetic equator ( $\pm x$ direction) in all cases. At the injection source, the magnetic flux density is $B_{i n j}=10 \mu \mathrm{T}$. Four kinetic $\beta_{i n j}$ values defined as Eq. (10) at the plasma injection source are used, and they are high in order of Case 1, Case 2, Case 3 and Case 4. That is, Case 1 indicates the case for the highest kinetic $\beta_{i n j}$ plasma injection and Case 4 indicates the case for the lowest kinetic $\beta_{i n j}$ plasma injection. The thermal $\beta_{i n j}$ (the ratio of the plasma pressure to the 
magnetic pressure at the injection source) is a constant at $8.9 \times 10^{-6}$ and the thermal $\beta_{i n j}$ is smaller than the kinetic $\beta_{i n j}$ in all cases.

$$
\beta_{i n j}=\left(\frac{1}{2} N_{i n j} m_{i} V_{i n j, i}^{2}+\frac{1}{2} N_{i n j} m_{e} V_{i n j, e}^{2}\right) /\left(\frac{B_{i n j}^{2}}{2 \mu_{0}}\right)
$$

Simulation results are shown in Fig. 11, in which the coil magnetic moment is set parallel to the solar wind direction $\left(\alpha=0^{\circ}\right)$. The thrust generated by MPS has a maximum value at $\beta_{i n j} 2 \times 10^{-3}$ as shown in Fig. 11a. This feature agrees with the past research by MHD simulation performed by Nishida et al. [4], that is, the thrust becomes smaller when the plasma with too large kinetic $\beta_{i n j}$ is injected. Despite the fact that thrust of MPS by MHD simulation becomes approximately zero in the case of high- $\beta$ plasma injection, the thrust of MPS by Full-PIC simulation is larger than the thrust of magnetic sail $\left(F_{M P S}>F_{m a g}\right)$ in all cases. This is because the termination shock, which prevents the momentum of the solar wind from transmitting to the spacecraft, observed in MHD simulation becomes weaker when considering the finite Larmor effect of particles in Full-PIC simulation. In addition, the thrust of MPS by Full-PIC simulation is approximately proportional to the cross-sectional area of magnetosphere when comparing Fig. 11a (thrust of MPS) and Fig. 11c (cross-sectional length of magnetosphere).

In the following, thrust characteristics of MPS are discussed associated with the flow and field structures in Fig. 10. The thrust force of $20 \mu \mathrm{N} / \mathrm{m}$ is obtained in Case 1, which is 3.4 times larger than thrust without plasma injection, $5.8 \mu \mathrm{N} / \mathrm{m}$. On the contrary, thrust obtained in Case 4 is $8.0 \mu \mathrm{N} / \mathrm{m}$ and an increase of thrust by plasma injection is only $38 \%$. Figure 12 shows the density distribution of ion (a, d, g), the distribution of local kinetic $\beta(b, e, h)$ and the current density distribution (c, f, i) of Case 1, Case 4 and the magnetic sail, respectively. The clear density gap at the magnetopause of MPS becomes ambiguous in Fig. 12a in comparison with that at the magnetic sail's magnetopause in Fig. 12g. In contrast, the structure of magnetopause current is rather clear and the cross-sectional length of the magnetosphere in Case 1 is expanded from $1300 \mathrm{~m}$ (magnetic sail, Fig. 12i) to $3300 \mathrm{~m}$ (MPS, Fig. 12c). Note that $10 \%$ error in cross-sectional length is expected because of the broad magnetopause. From the above discussion, it is indicated that the expanded magnetospheric size greatly contributes to obtain a large thrust increment. Large magnetic field inflation in Case 1 is realized by the condition where electrons are magnetized and ions are not magnetized. Using the plasma parameters of the injected plasma, the Larmor radius of ion $\left(r_{i L} \sim 50 \mathrm{~km}\right)$ is very large $\left(r_{i L}>B / \nabla B\right)$ and the Larmor radius of electron $\left(r_{e L} \sim 2 \mathrm{~m}\right)$ satisfies the condition of $r_{e L}<B / \nabla B$. Here, the characteristic length of magnetic field $B / \nabla B$ at the source is approximately calculated as $B / \nabla B \sim R_{\text {plasma }} / 2=75 \mathrm{~m}$. The plasma flow 
toward the injection direction $( \pm x)$ expands the magnetic field since the magnetized electrons move with ions to satisfy the charge neutrality. On the contrary, in Case 4, only a slight magnetospheric inflation is obtained, that is, from $1300 \mathrm{~m}$ (magnetic sail, Fig. 12i) to $1400 \mathrm{~m}$ (MPS, Fig. 12f). The Larmor radius of injected ions $\left(r_{i L} \sim 0 \mathrm{~m}\right)$ and electrons $\left(r_{e L} \sim 2 \mathrm{~m}\right)$ satisfy the conditions of $r_{i L}<B / \nabla B$ and $r_{e L}<B / \nabla B$, respectively, and injection plasma is strongly magnetized. The magnetized plasma, especially electron, induces the large diamagnetic current in the same direction of the coil current in the region around the injection source, where the density of plasma is very high, as shown in Fig. 12f. The diamagnetic current causes the magnetospheric inflation in Case 4. Here, we note that the contour level of the current density distributions in Fig. $12 \mathrm{f}$ is 10 times larger than the contour level in Fig. 12c. In addition, although there are streamlines that penetrate into the magnetosphere of the magnetic sail (Fig. 12g), all streamlines are dammed in Case 4 (Fig. 12d) by the mirror magnetic field. As a result, thrust of MPS in Case 4 is also increased by a plasma injection.

Figures $12 \mathrm{~b}$ and $12 \mathrm{e}$ represent the distribution of the local kinetic $\beta$ in Case 1 and Case 4 , respectively. The local kinetic $\beta$ is calculated by using the density and the mean velocity of the particles at the local point. At the magnetopause, pressure equilibrium between the solar wind dynamic pressure and the magnetic pressure is approximately established and the local kinetic $\beta$ approximately becomes unity. In Case 1 and 4 , as the magnetosphere is inflated, the boundary of $\beta=1.0$ is also inflated from the case of magnetic sail (Fig. 12h). Around the plasma injection source of Case 1 , the local kinetic $\beta$ becomes higher than unity; in this case, the injected particles cannot accumulate inside the magnetosphere and the plasma flows out toward $\pm x$. On the contrary, in Case 4 , the local kinetic $\beta$ is lower than unity around the injection source; in this case the injected plasma is strongly magnetized and the plasma flow toward $\pm x$ does not occur. As a result, the very high-density region is formed around the injection source and the larger diamagnetic current is induced in Case 4 as the above discussion.

So far, it is successfully shown that thrust by magnetic sail can be significantly increased by MPS. However, it is probable that significant amount of thrust can be obtained by collimating a released plasma jet from spacecraft. To evaluate true "thrust gain" by MPS, thrust gain is evaluated based on Eq. (11). In this equation, $F_{\text {mag }}$ means thrust by magnetic sail and equivalent thrust $F_{j e t}$ indicates thrust by injected plasma. $F_{j e t}$ is calculated as Eq. (12) assuming that injected plasma is collimated to one direction and listed in Table 7.

$$
\text { Thrust gain }=\frac{F_{M P S}}{F_{\text {mag }}+F_{\text {jet }}}
$$




$$
F_{j e t}=N_{i n j} m_{i} Q V_{i n j, i}+N_{i n j} m_{e} Q V_{i n j, e}+N_{i n j} k_{B} T_{i} S+N_{i n j} k_{B} T_{e} S
$$

The first and second term in Eq. (12) represent the momentum thrust of ion and electron, respectively. The third and fourth term represent the static pressure thrust of ion and electron, respectively. Here, $S$ is assumed to be $4 \mathrm{~d} x$, the cross section of four injection sources. In Case 1 , the thrust gain is calculated as only 0.05 since the large injection velocity of ions increases $F_{\text {jet }}$. In Case 4, the thrust gain is calculated as 1.3 . Thus, the thrust of MPS can be enhanced significantly by the low kinetic $\beta_{i n j}$ plasma injection. The inflation by the diamagnetic current (Case 4$)$ expands the magnetic field efficiently than the inflation by the frozen-in of plasma (Case 1).

In addition, as shown in Fig. 11b, the largest thrust gain can be obtained in Case 2. We note that the parameters in Case 2 indicate the moderately low kinetic $\beta_{i n j}$ plasma injection nevertheless the electron has large injection velocity since the electron mass is 1836 times lighter than ion mass when using the realistic electron-ion mass ratio. The ion velocity and the electron velocity in this case satisfy $r_{i L}(\sim 50 \mathrm{~m}) \sim B / \nabla B$ and $r_{e L}(\sim 30 \mathrm{~m})<B / \nabla B$, respectively. Under this condition, magnetized plasma induces the diamagnetic current, which mainly consists of the drift motion of electron in magnetosphere since the drift velocity is proportional to the square of the injection velocity $\left(V_{i n j, i}<<V_{i n j,}\right.$ e). In addition, the ions raise the flow toward the injection direction $( \pm x)$ around the injection source. Consequently, in Case 2 the strong and widespread diamagnetic current is formed around the coil as shown in Fig. 13 by the solid line. The induced current near the coil enhances the original magnetic field and thus the magnetosphere becomes larger. Figure 14 shows the ion density distribution in Case 2 for both the parallel case $\left(\alpha=0^{\circ}\right)$ and the perpendicular case $\left(\alpha=90^{\circ}\right)$. As compared with Fig. 9, the magnetosphere is significantly inflated by the plasma injection. The thrust in the perpendicular case is calculated as $15 \mu \mathrm{N} / \mathrm{m}$ that is 7.5 times larger than the magnetic sail thrust of 2.0 $\mu \mathrm{N} / \mathrm{m}$. The thrust in the parallel case is calculated as $26 \mu \mathrm{N} / \mathrm{m}$ that is 4.4 times larger than the magnetic sail thrust, $5.8 \mu \mathrm{N} / \mathrm{m}$. The thrust gains in the above two cases are also calculated as 1.9 and 2.4 , respectively.

Time history of the magnetospheric inflation in Case $2\left(\alpha=90^{\circ}\right)$ is shown in Fig. 15 . As the total amount of the injected plasma trapped in the magnetosphere increases, the magnetospheric size becomes larger. The solar wind flow is also bended by the enhanced magnetic field and loses its momentum to produce the larger thrust. The onedimensional profiles of the magnetic flux density and the ion density along $z$-axis $(x=0 \mathrm{~m})$ are shown in Fig. 16 . The magnetospheric size of the MPS becomes 3 times larger than the original magnetospheric size of the magnetic sail as shown in Fig. 14a. The higher density around the source represents the injected plasma trapped by the magnetic 
field. The diamagnetic current is formed around $z=150 \mathrm{~m}$ and $z=-150 \mathrm{~m}$. The current enhances the magnetic field outside of the current and reduce the magnetic field inside of the current as shown in Fig. 16b.

Table 6 Magnetic sail design parameters

\begin{tabular}{cc}
\hline \hline Magnetic sail parameters & \\
\hline Coil distance $r_{\text {coil }}$ & $75 \mathrm{~m}$ \\
Coil current $\times$ Turn $I_{\text {coil }}$ & $4 \times 10^{3}$ A Turn \\
Theoretical magnetospheric size $L$ & $2.2 \times 10^{3} \mathrm{~m}$ \\
\hline \hline
\end{tabular}

Table 7 Magneto Plasma Sail design parameters

\begin{tabular}{|c|c|c|c|c|}
\hline Plasma injection parameters & Case 1 & Case 2 & Case 3 & Case 4 \\
\hline Plasma density $N_{i n j}\left[\mathrm{~m}^{-3}\right]$ & $5.0 \times 10^{7}$ & $5.0 \times 10^{7}$ & $5.0 \times 10^{7}$ & $5.0 \times 10^{7}$ \\
\hline Ion velocity $V_{i n j, i}[\mathrm{~m} / \mathrm{s}]$ & $5.0 \times 10^{6}$ & $5.0 \times 10^{4}$ & $5.0 \times 10^{5}$ & 0 \\
\hline Ion temperature $T_{i n j, i}[\mathrm{eV}]$ & 100 & 100 & 100 & 100 \\
\hline Electron velocity $V_{i n j, e}[\mathrm{~m} / \mathrm{s}]$ & $5.0 \times 10^{6}$ & $5.0 \times 10^{7}$ & $5.0 \times 10^{5}$ & $5.0 \times 10^{6}$ \\
\hline Electron temperature $T_{i n j, e}[\mathrm{eV}]$ & 100 & 100 & 100 & 100 \\
\hline Kinetic $\beta_{i n j}$ & $4.1 \times 10^{-2}$ & $2.2 \times 10^{-3}$ & $4.1 \times 10^{-4}$ & $2.2 \times 10^{-5}$ \\
\hline Flow rate $Q\left[\mathrm{~m}^{2} / \mathrm{s}\right]$ & $9.0 \times 10^{8}$ & $9.0 \times 10^{8}$ & $9.0 \times 10^{8}$ & $9.0 \times 10^{8}$ \\
\hline Equivalent thrust $F_{j e t}[\mathrm{~N} / \mathrm{m}]$ & $3.8 \times 10^{-4}$ & $5.8 \times 10^{-6}$ & $3.8 \times 10^{-5}$ & $2.0 \times 10^{-7}$ \\
\hline
\end{tabular}




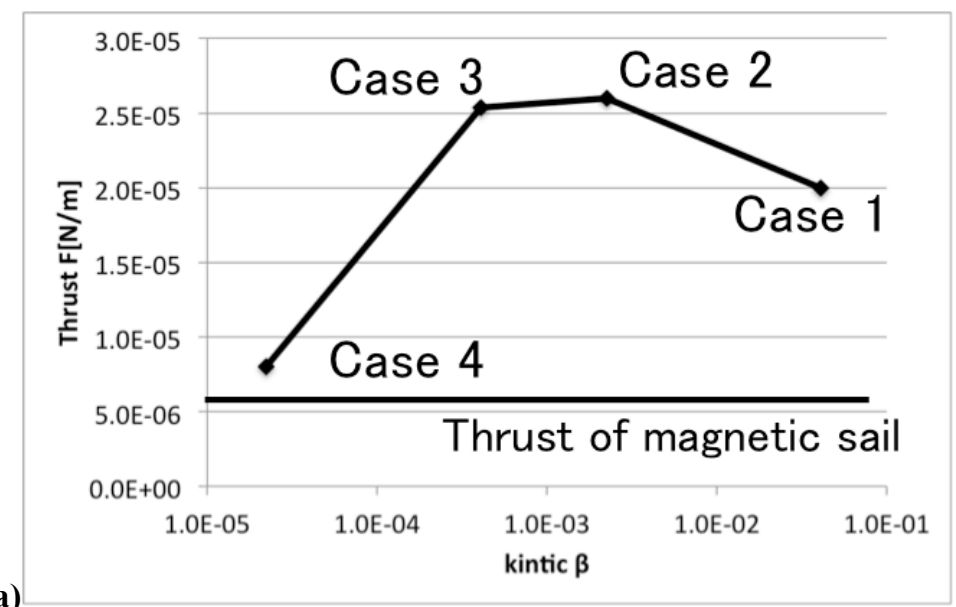

a)

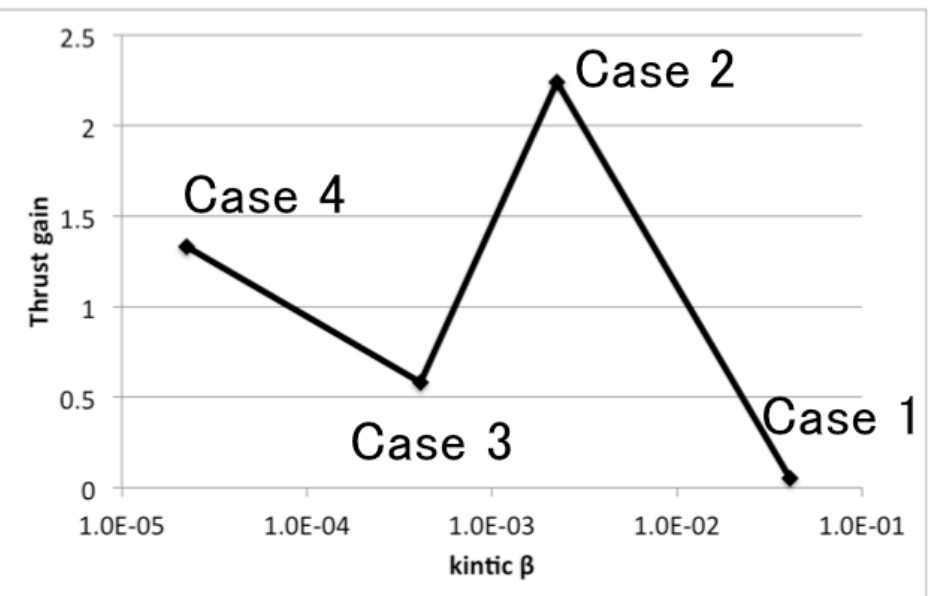

b)

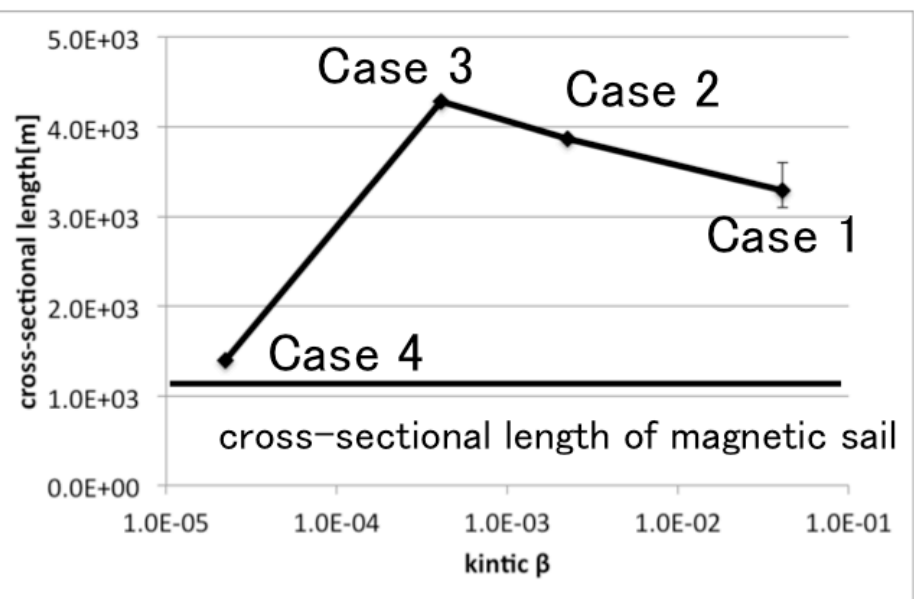

c)

Fig. 11 a) thrust of MPS $F_{M P S}$, b) thrust gain $F_{M P S} /\left(F_{m a g}+F_{j e t}\right)$ and c) cross-sectional length of the magnetosphere obtained by the various kinetic $\beta_{i n j}$ plasma injections $\left(L=2200 \mathrm{~m}, \alpha=0{ }^{\circ}\right)$. Thrust and cross-sectional length of the magnetic sail are $5.8 \mu \mathrm{N} / \mathrm{m}$ and $1300 \mathrm{~m}$, respectively. 
a)

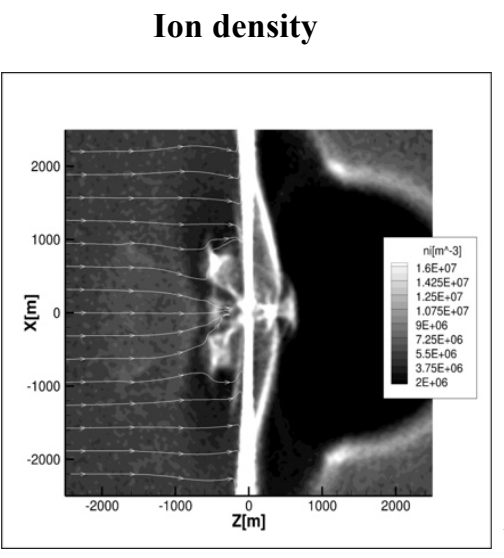

d)
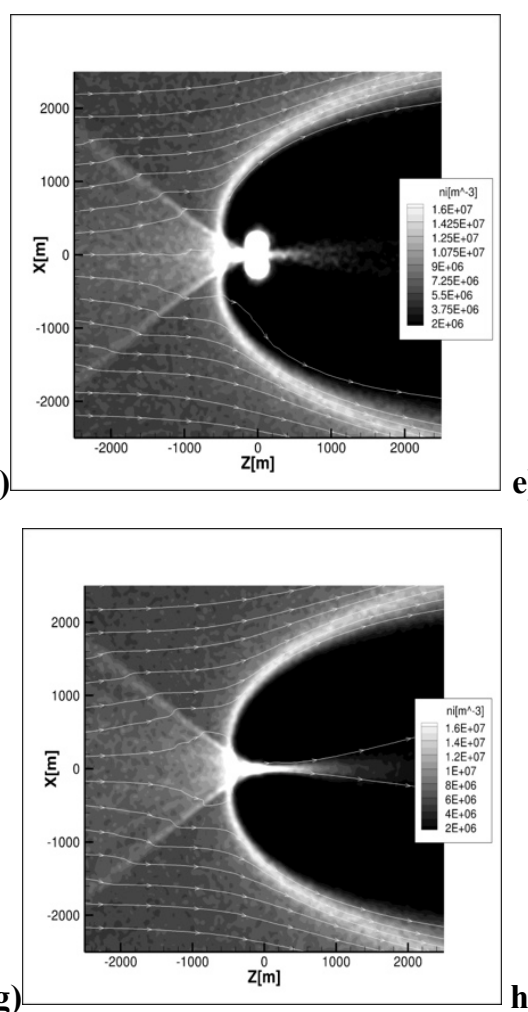

local kinetic $\beta$

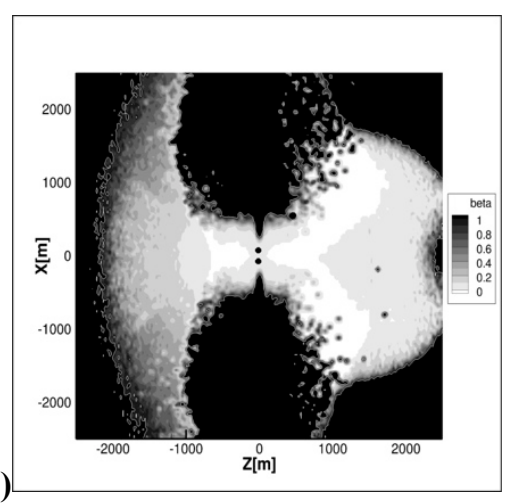

b)
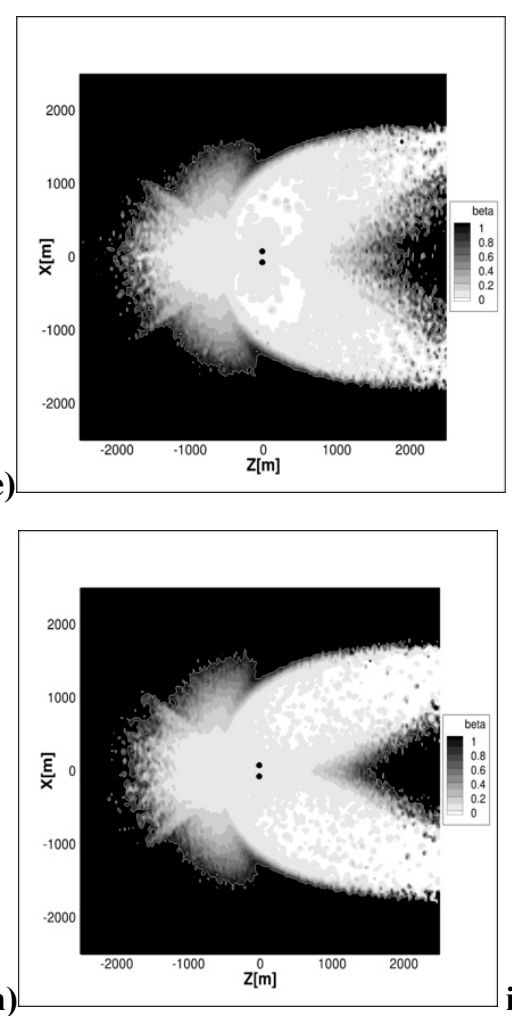

c)
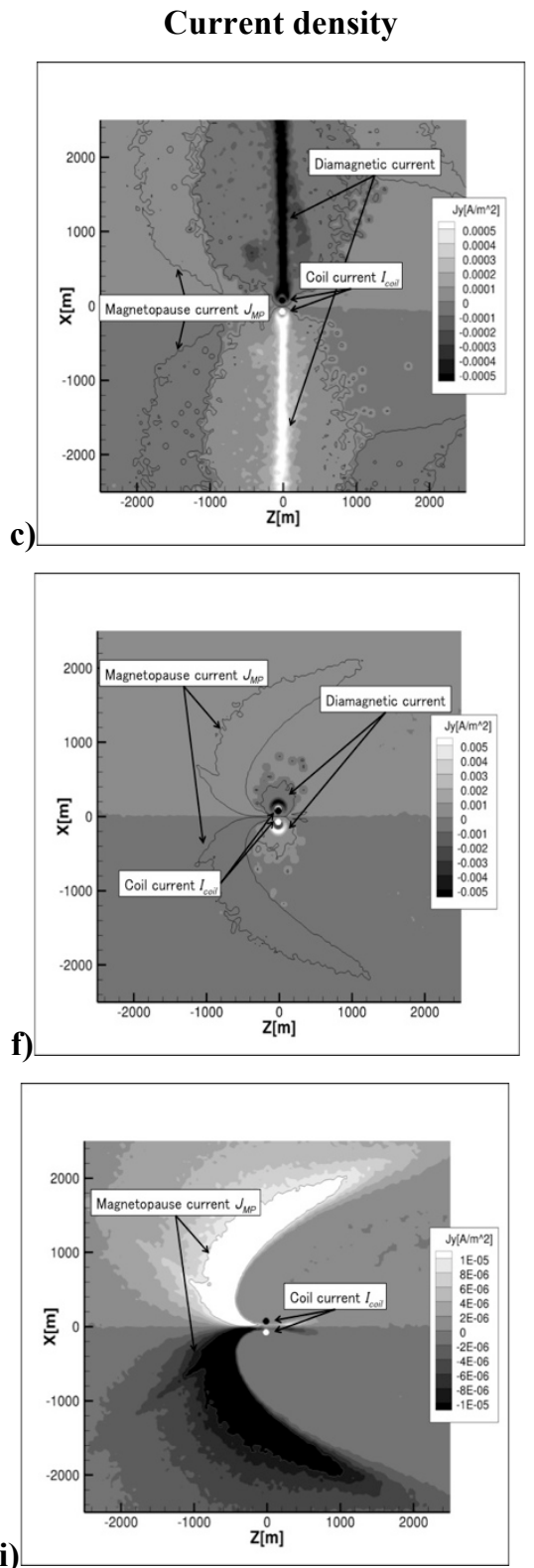

Fig. 12 Distribution of ion density, local kinetic $\beta$ and current denisty distribution: a, b, c) result for Case 1 (MPS) with the high kinetic $\beta_{i n j}$ plasma injection, $\left.d, e, f\right)$ result for Case 4 (MPS) with the low $\beta_{i n j}$ plasma injection and $\mathrm{g}, \mathrm{h}$, i) result for the original magnetic sail $\left(L=2200 \mathrm{~m}, \alpha=0{ }^{\circ}\right)$. The contour lines of $\pm 10 \mu \mathrm{A} / \mathrm{m}^{2}$ are drawn in the current density distribution to show the magnetopause current. 


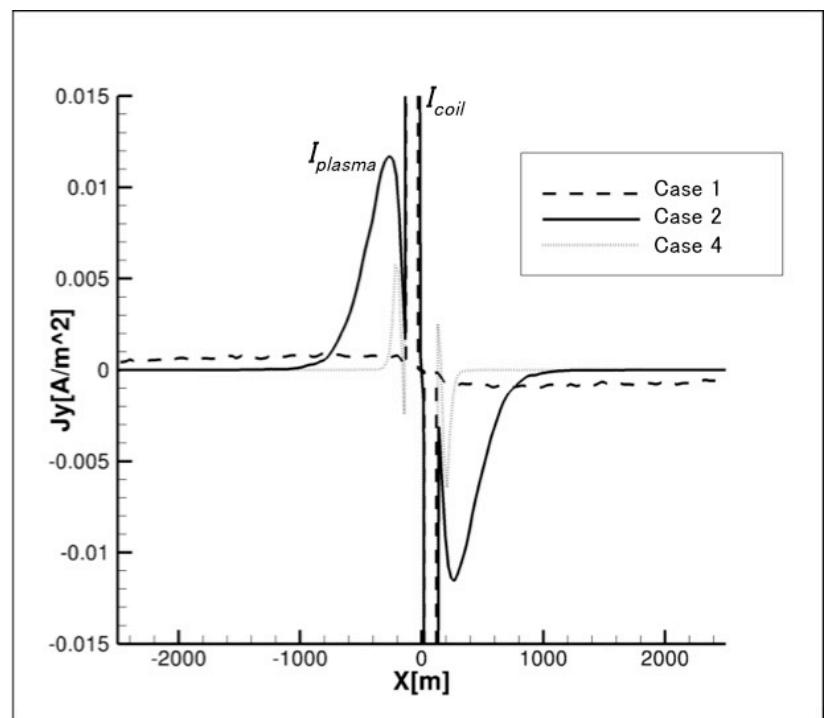

Fig. 13 Current density distribution along $x$-axis $(z=0 \mathrm{~m})$ in Case 1 (dashed), Case 2 (solid), case 4 (dotted). The current widely spreads by the high kinetic $\beta_{i n j}$ plasma injection and the current flows locally by the low high kinetic $\beta_{i n j}$ plasma injection. The strong and widespread diamagnetic current is formed by Case 2.
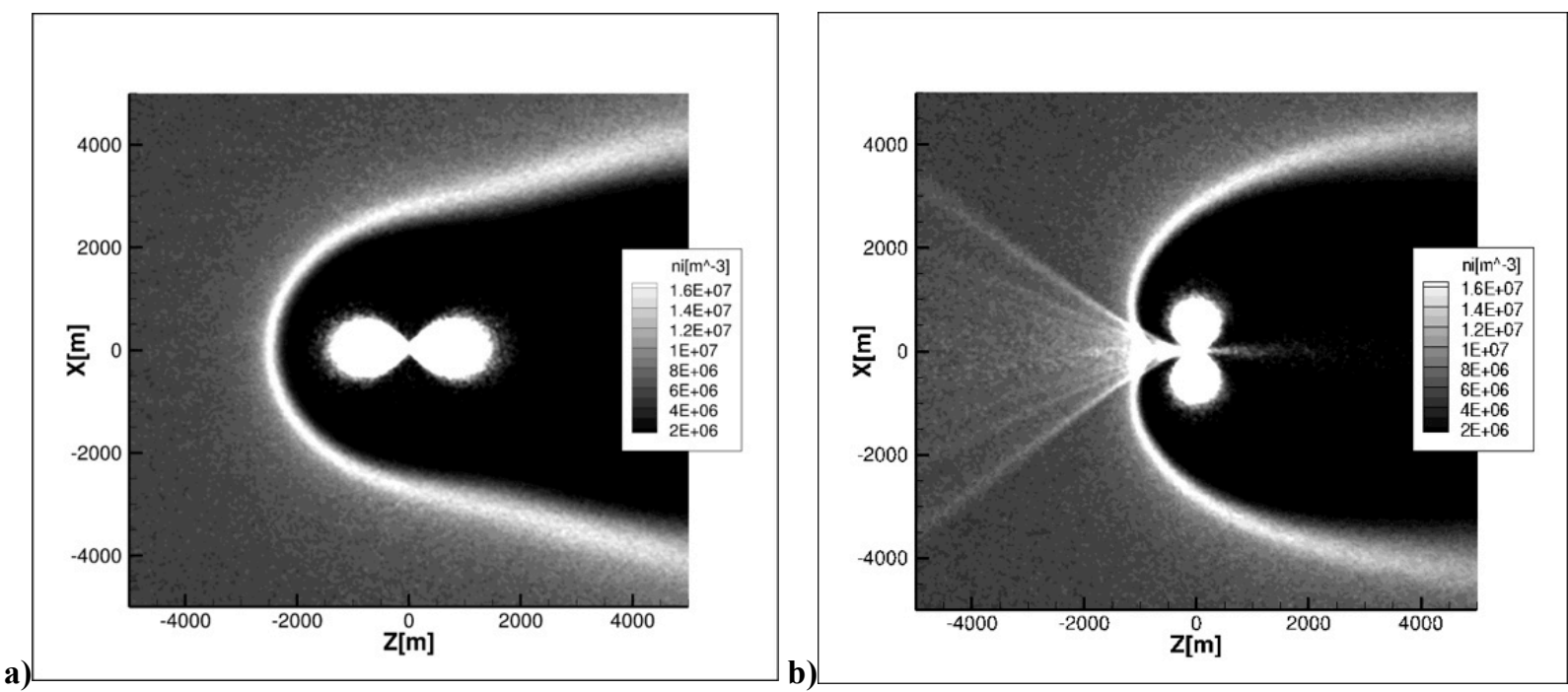

Fig. 14 Ion density distribution in Case 2 (MPS, $L=2200 \mathrm{~m})$ : a) perpendicular case $\left(\alpha=90^{\circ}\right)$ and b) parallel case $(\alpha=0$ ). 


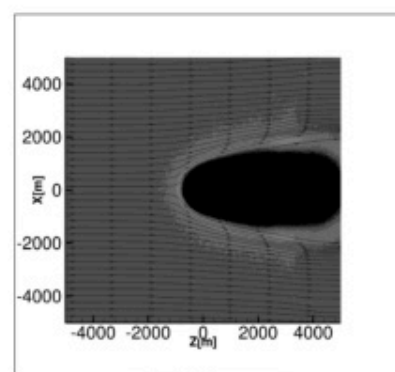

$\mathrm{t}=7.5 \mathrm{~ms}$

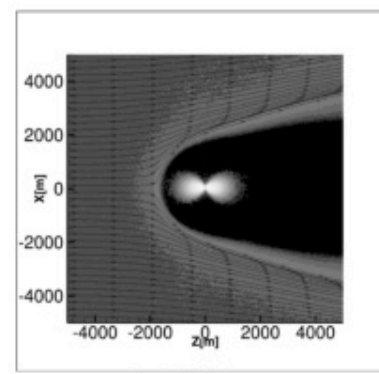

$\mathrm{t}=30.0 \mathrm{~ms}$

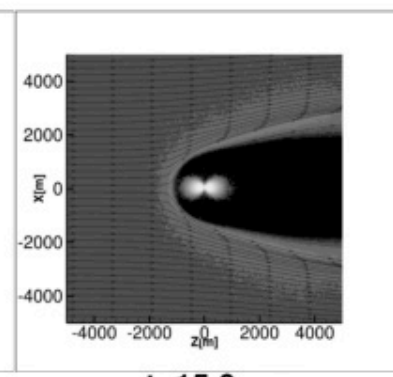

$\mathrm{t}=15.0 \mathrm{~ms}$

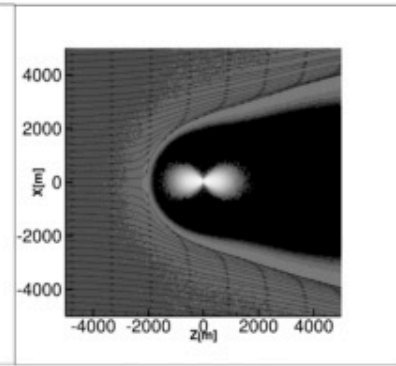

$\mathrm{t}=37.5 \mathrm{~ms}$

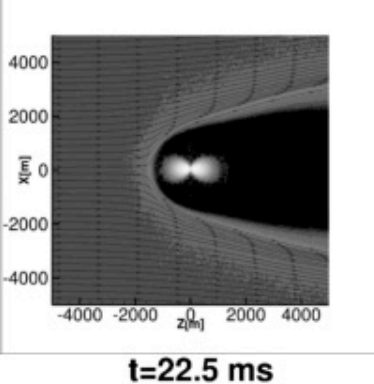

$\mathrm{t}=22.5 \mathrm{~ms}$

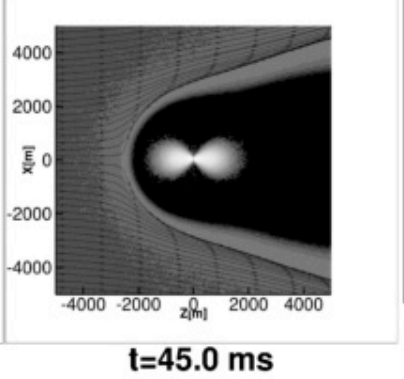

ni[m^-3]

$5.3 \mathrm{E}+09$

$3.0 \mathrm{E}+09$

$1.7 \mathrm{E}+09$

$9.8 \mathrm{E}+08$

$5.6 \mathrm{E}+08$

$3.2 \mathrm{E}+08$

$1.8 \mathrm{E}+08$

$1.0 \mathrm{E}+08$

$5.9 \mathrm{E}+07$

$3.3 \mathrm{E}+07$

$1.9 \mathrm{E}+07$

$1.1 \mathrm{E}+07$

$6.2 \mathrm{E}+06$

$3.5 \mathrm{E}+06$

$2.0 \mathrm{E}+06$

Fig. 15 Time history of the magnetospheric inflation in Case $2\left(L=2200 \mathrm{~m}, \alpha=90^{\circ}\right)$. As time passing, the magnetosphere size of MPS becomes larger. The plasma injection starts after $t=7.5 \mathrm{~ms}$. 

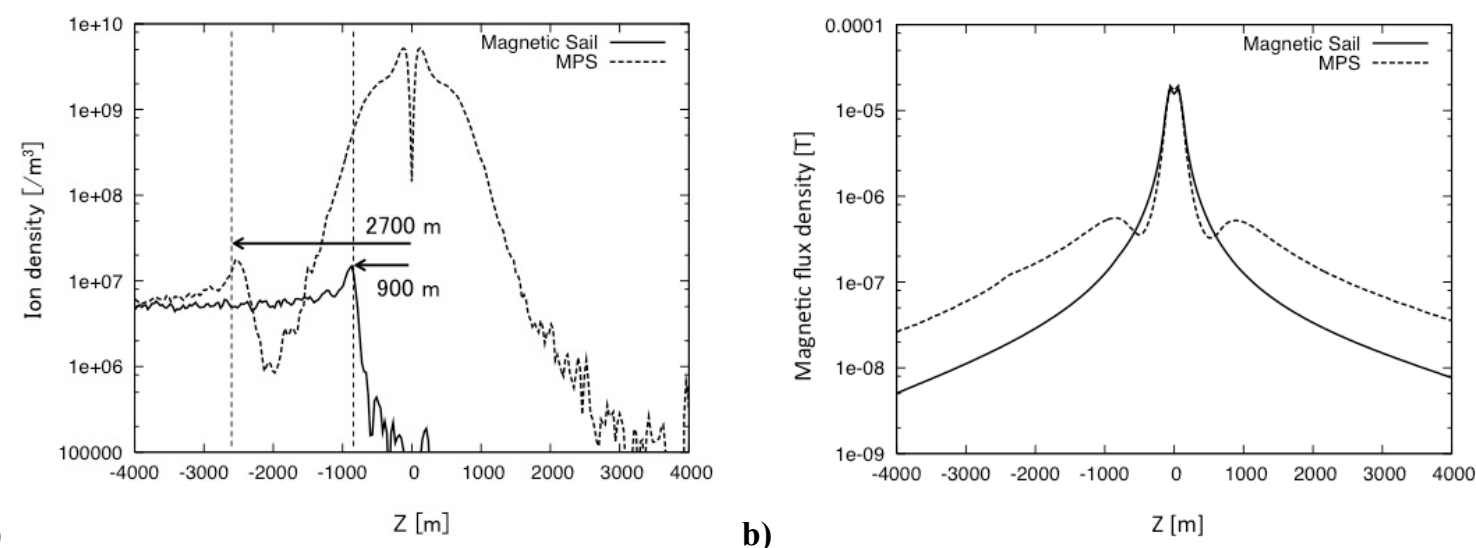

b)

Fig. 16 Density distribution of ion and magnetic flux density along $z$-axis $(x=0 \mathrm{~m})$ for the perpendicular case $\left(\alpha=90^{\circ}\right.$, Case 2). a) represents ion density of magnetic sail (solid line) and MPS (dotted line). b) represents magnetic flux density of magnetic sail (solid line) and MPS (dotted line).

\section{B. Estimation of thrust increase by the diamagnetic current}

By the plasma injection, the plasma current is formed around the injection source as shown in Figs. 12 and 13. The plasma current diameter is small compared to the magnetospheric size for the low kinetic $\beta_{i n j}$ plasma injection case such as Case 2. Hence, a thin wire current model in Fig. 17 is introduced to evaluate an increase in the magnetic moment by a diamagnetic current. Based on this simple $2 \mathrm{D}$ assumption, the diamagnetic plasma current is represented by the line current $I_{\text {plasma }}$, which flows in the same direction with the coil current $I_{\text {coil }}$. The thin wire approximation is expected to be sufficient since the diameter of the plasma current is small in comparison with the coil distance. The force applied on the plasma current $I_{\text {plasma }}$ is simply obtained as Eq. (13) by calculating the magnetic field generated by the each thin wire current. The first term of Eq. (13) is an attracting force between $I_{\text {coil }}$ and $I_{\text {plasma }}$. The second and the third term represent the repulsive force acting on $I_{\text {plasma }}$ by $I_{\text {coil }}$ and $I_{\text {plasma }}$, respectively. When the forces are balanced $\left(F_{\text {plasma }}=0\right)$ and the plasma current becomes stable, the ratio of the coil current $I_{\text {coil }}$ and the plasma current $I_{\text {plasma }}$ is calculated as Eq. (14). The total magnetic moment by the coil current and the plasma current is calculated as Eq. (15) by using Eq. (14). The figures in parenthesis mean the scaling factor of the magnetic moment. When parameters used in the simulation $\left(R_{\text {coil }}=75 \mathrm{~m}\right.$ and $\left.R_{\text {plasma }}=150 \mathrm{~m}\right)$ are substituted, the factor becomes 6.3. That is, the diamagnetic current is able to enhance the original magnetic moment 6.3 times larger. Then, the magnetospheric size by the MHD approximation is inflated 2.5 times since $L$ is proportional to square root of the magnetic moment as Eq. (2). When the original magnetospheric size is set as $L=2200 \mathrm{~m}$, the magnetospheric size by the MHD approximation is expected to be expanded up to $L=5500 \mathrm{~m}$. The thrust levels of $15 \mu \mathrm{N} / \mathrm{m}$ in the perpendicular case and of $30 \mu \mathrm{N} / \mathrm{m}$ in the parallel case are expected by linearly extrapolating the thrust 
characteristics represented in Fig. 8a in section III to $L=5500 \mathrm{~m}$. Compared to the simulation result in section IV A, the magnetospheric size is inflated three times since the cross-sectional length of the magnetosphere and the theoretical $L$ have the relation as shown in Fig. 8c and the thrust of $15 \mu \mathrm{N} / \mathrm{m}$ and $26 \mu \mathrm{N} / \mathrm{m}$ are obtained in perpendicular case and parallel case, respectively. The thrust of MPS obtained by Full-PIC simulation with the low kinetic $\beta_{i n j}$ plasma injection can be approximately estimated by the simple assumptions about the diamagnetic current and the thrust characteristics of magnetic sail.

$$
\begin{gathered}
F_{\text {plasma }}=\frac{\mu_{0}}{2 \pi}\left(-\frac{I_{\text {coil }} I_{\text {plasma }}}{R_{\text {plamsa }}-R_{\text {coil }}}+\frac{I_{\text {coil }} I_{\text {plasma }}}{R_{\text {plamsa }}+R_{\text {coil }}}+\frac{I_{\text {plasma }} I_{\text {plasma }}}{2 R_{\text {plamsa }}}\right) \\
\frac{I_{\text {plasma }}}{I_{\text {coil }}}=\frac{4 R_{\text {coil }} R_{\text {plasam }}}{R_{\text {plasma }}^{2}-R_{\text {coil }}^{2}} \\
M=R_{\text {coil }} I_{\text {coil }}+R_{\text {plasma }} I_{\text {plasma }}=R_{\text {coil }} I_{\text {coil }}\left(1+\frac{4 R_{\text {plasma }}^{2}}{R_{\text {plasma }}^{2}-R_{\text {coil }}^{2}}\right)
\end{gathered}
$$

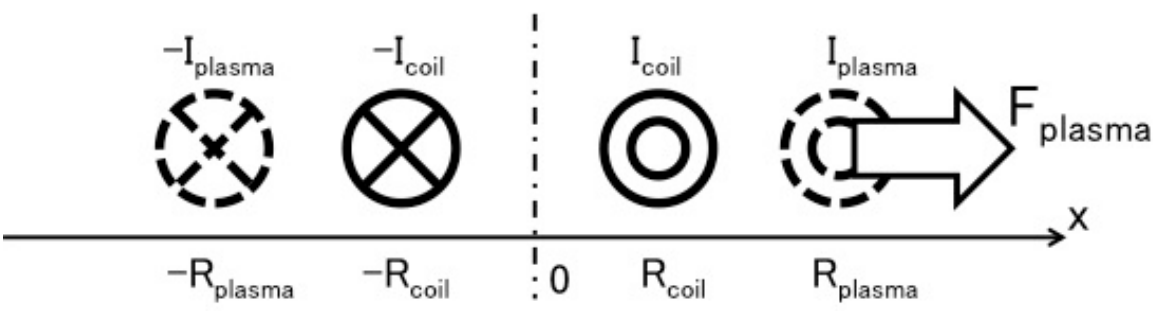

Fig. 17 Simplest current model of MPS. Along the $x$-axis, finite opposite currents are defined. The current at $x=+R_{\text {coil }}$ and $x=-R_{\text {coil }}$ represents the coil and the current at $x=+R_{\text {plasma }}$ and $x=-R_{\text {plasma }}$ represents the plasma current induced by plasma injection. The double circle means upward current perpendicular to the plane and the cross-circle means downward current perpendicular to the plane. The current is assumed to be a thin wire for simplicity. 


\section{Thrust performance of a MPS on the electron inertial scale}

Finally, we examined the thrust performance of MPS spacecraft based on the above analysis result. However, the thrust performance shown here may differ from that of the actual spacecraft in three-dimension since the analysis is performed in two-dimension. We chose the case 2 for the MPS spacecraft model since the largest thrust is obtained. The details of the superconducting material assumed here are shown in Table 8 . The weight of the superconductive coil per unit length is calculated as $0.158 \mathrm{~kg} / \mathrm{m}\left(4.3 \mathrm{~mm} \times 0.23 \mathrm{~mm} \times 10 \mathrm{~g} / \mathrm{cm}^{3} \times 4 \times 10^{3}\right.$ ATurn $/ 500$ A $\left.\times 2\right)$ without considering the cooling system and the bus system. As a result, the thrust-mass ratio of the MPS is calculated as 0.16 $\mathrm{mN} / \mathrm{kg}(26 \mu \mathrm{N} / \mathrm{m} / 0.158 \mathrm{~kg} / \mathrm{m})$. This is similar to the value theoretically predicted by Cattell et al. [10] $(0.23 \mathrm{mN} / \mathrm{kg}$, 3-ton superconductive coil and thrust generation of $700 \mathrm{mN}$ ). The thrust-mass ratio of the MPS is also competitive with that of other sail type propulsion system such as solar sail $(0.07 \mathrm{mN} / \mathrm{kg}$ of IKAROS, here only mass of the reflection mirror is considered). If compared with ion engines $(0.40 \mathrm{mN} / \mathrm{kg}$ of HAYABUSA and $1.9 \mathrm{mN} / \mathrm{kg}$ of Deep Space 1, here dry mass of the thrust system without the bus system and the mission equipment), the thrust-mass ratio of MPS is still an order of magnitude smaller. However, by the improvement of the superconductive technology and the optimal design of the superconductive coil to magnetic sail [21, 22], it is expected that the thrust-mass ratio should be improved by 2 5 times compared with the assumption of this study.

In addition, by considering the flow rate $Q$ and the plasma density of injection $N_{i n j}$, the specific impulse of this MPS is calculated as $I_{s p}=38000 \mathrm{~s}$. The specific impulse of the MPS is much higher than that of other electric propulsions (3200 s of HAYABUSA and 1000-3000 s of Deep Space 1). This is because MPS converts not the equipped propellant but the momentum of the solar wind to the thrust force. The high specific impulse will make it possible to reduce the wet mass of the spacecraft.

The thrust performance of MPS is thus a very attractive for the deep space exploration. It remains to be analyzed whether the similar thrust performance can be obtained in the three-dimensional model and what kind of missions is suitable for MPS with the high specific impulse.

Table 8 Details of superconductive wire material

\begin{tabular}{cc}
\hline \hline Type & DI-BSCCO Type H \\
Width & $4.3 \mathrm{~mm}$ \\
Thickness & $0.23 \mathrm{~mm}$ \\
Density & $10 \mathrm{~g} / \mathrm{cm}^{3}$ \\
Critical Current & $500 \mathrm{~A}$ \\
\hline \hline
\end{tabular}




\section{Conclusion}

We performed two-dimensional Full-PIC simulations for magnetic sail and magneto plasma sail (MPS) with very small artificial magnetosphere in which electron kinetics such as the electron's finite Larmor radius effect and charge separation between ion and electron become significant. As a result, the charge-separated and smaller magnetosphere compared with MHD approximation was formed and it was found that the Larmor motion of electron affects the magnetosphere formation on such a small magnetosphere. In addition, it was revealed by the simulations with 16 magnetospheric sizes from $380 \mathrm{~m}$ to $4300 \mathrm{~m}$ that the thrust of small magnetic sail differently depends on the cross-sectional length of the magnetosphere and the magnetic field property such as the mirror magnetic field according to the attack angle $\alpha$. MPS simulations also revealed that MPS could inflate the magnetosphere and the thrust can be improved by taking particle kinetics into account. MPS thrust was calculated by using four characteristic parameter sets depending on the plasma injection energy equivalent to not only the frozen-in concept but also the newly proposed ring-current concept. As a result, the diamagnetic current induced by moderately low kinetic $\beta\left(\sim 10^{-3}\right)$ plasma injection, which flows in the same direction as the onboard coil current, enhanced the original magnetic field successively. The thrust of the MPS $(15 \mu \mathrm{N} / \mathrm{m}$ in perpendicular case and 26 $\mu \mathrm{N} / \mathrm{m}$ in parallel case) becomes up to 7.5 times larger than the original thrust of the magnetic sail $(2.0 \mu \mathrm{N} / \mathrm{m}$ and 5.8 $\mu \mathrm{N} / \mathrm{m}$ ). The thrust gain defined as "MPS thrust / (Magnetic sail thrust + plasma injection thrust)" is calculated as approximately two times in both perpendicular case and parallel case. Finally, it was revealed that the thrust-mass ratio of the MPS is $0.16 \mathrm{mN} / \mathrm{kg}$ and the higher specific impulse, $38000 \mathrm{~s}$, than the existing electric propulsion systems can be obtained.

By the two-dimensional Full-PIC simulation, the fundamental mechanism of the thrust generation of magnetic sail and MPS with the electron inertial scale magnetospheres was revealed and it was demonstrated that the concept of MPS using the newly proposed ring-current and low kinetic $\beta$ plasma injection concept is attractive as a deep space propulsion system. In future work, three-dimensional Full-PIC simulation should be performed to obtain the thrust level of MPS based on the actual spacecraft model and to design a demonstrator spacecraft. By threedimensional analysis, the exact influence of IMF such as a magnetic reconnection will be taken into consideration. 


\section{Acknowledgments}

A part of computation in the present study was performed with K computer at RIKEN (Number hp120084), the Kyoto-daigaku Denpa-kagaku Keisanki-jikken (KDK) system of Research Institute for Sustainable Humanosphere (RISH) at Kyoto University and JAXA supercomputing system (JSS) as a collaborative research project. We gratefully acknowledge the support and advice of the members of the Magneto Plasma Sail research group in Japan. This work also has been supported by Core Research for Evolutional Science and Technology (CREST) of Japan Science and Technology Agency (JST) and JSPS KAKENHI Grant Number 241626.

\section{References}

[1] Zubrin, R. M., and Andrews, D. G., "Magnetic Sails and Interplanetary Travel," Journal of Spacecraft and Rockets, Vol. 28, No. 2, 1991, pp.197-203.

[2] Winglee, R. M., Slough, J., Ziemba, T., and Goodson, A., "Mini-Magnetospheric Plasma Propulsion: Trapping the Energy of the Solar Wind for Spacecraft Propulsion,” Journal of Geophysical Research, Vol. 105, 2000, pp. 21067-21077.

[3] Nishida, H. and Nakayama, Y., "Two-dimensional Magnetohydrodynamic Simulation of a Magnetic Sail," Journal of Spacecraft and Rockets, Vol. 43, No. 3, 2006, pp. 667-672.

[4] Nishida, H., Funaki, I., Ogawa, H. and Inatani, Y., "MHD Analysis on Propulsive characteristics of Magneto Plasma Sail," 30th International Electric Propulsion Conference, Florence, Italy, IEPC-2007-195, Sep, 2007.

[5] Nishida, H. and Funaki, I., "Analysis of Thrust Characteristics of a Magnetic Sail in Magnetized Solar Wind," Journal of Propulsion and Power, Vol. 28, No. 3, 2012, pp. 336-642.

[6] Khazanov, G., Delamere, P., Kabin, K. and Linde, T. J.,” Fundamentals of the Plasma Sail Concept: Magnetohydrodynamic and Kinetic Studies,” Journal of Propulsion and Power, Vol. 21, No. 5, 2005, pp. 853-861.

[7] Winske, D. and Omidi, N., "Plasma expansion in the presence of a dipole magnetic field," Physics of Plasmas, Vol. 12, 2005, pp. 072514-1-12.

[8] Kajimura, Y., Funaki, I., Shinohara, I., Usui, H. and Yamakawa, H., “Thrust Evaluation of Magneto Plasma sail by Using Three-Dimensional Hybrid PIC Code," 46st AIAA/ASME/SAE/ASEE Joint Propulsion Conference and Exhibit, Nashville, Tennessee, AIAA-2010-6686, 2010.

[9] Moritaka, T., Usui, H., Nunami, M., Kajimura, Y., Nakamura, M. and Matsumoto, M., "Full Particle-in-Cell Simulation Study on Magnetic Inflation around a Magneto Plasma Sail,” Plasma Science IEEE Transactions on, Vol. 38, No. 9, 2010, pp. 2219-2228. 
[10] Cattell, C., Catto, P., Funsten, H., Garnier, D., Hershkwitz, N., Myers, R., Petschek, H. and Winske, D., "Physics and Technology of the Feasibility of Plasma Sails," Plasma Science and Fusion Center website: http://www.psfc.mit.edu/library1/catalog/reports/2000/05ja/05ja026/05ja026_full.pdf.

[11] Pfeiffer, M., Petkow, D., Herdich, G. and Fasoulas, S., "Assesment of a Numerical Approach Suitable for the M2P2 Problem," The Open Plasma Physics Journal, Vol. 4, 2011, pp. 24-33.

[12] Matumoto, H. and Omura, Y., Computer Space Plasma Physics, Terra Scientific Publishing Company, Tokyo, Japan, 1993, pp. 37-41.

[13] Nishida, H., Ogawa, H., Funaki, I., Fujita, K., Yamakawa, H., and Inatani, Y., "Verification of Momentum Transfer Process on Magnetic Sail Using MHD Model," 41st AIAA/ASME/SAE/ASEE Joint Propulsion Conference and Exhibit, Tucson, Arizona, Paper 2005-4463, Jul, 2005.

[14] Fujita, K., "Particle Simulation of Moderately Sized Magnetic Sails," Journal of Space Technology and Science, Vol. 20 , No. 2, 2005, pp. 26-31.

[15] Kajimura, Y., Usui, H., Funaki, I., Ueno, K., Nunami, M., Sinohara, I., Nakamura, N. and Yamakawa, H., "Hybrid Particlein-Cell Simulations of Magnetic Sail in Laboratory Experiment,” Journal of Propulsion and Power, Vol. 26, No. 1, 2010, pp. 159-166.

[16] Kajimura, Y., Funaki, I., Matsumoto, M., Shinohara, I., Usui, H. and Yamakawa, H., "Thrust and Attitude Evaluation of Magnetic Sail by Three-dimensional Hybrid Particle-in-Cell Code,” Journal of Propulsion and Power, Vol. 28, No. 3, 2012, pp. 652-663.

[17] Ashida, Y., Funaki, I., Yamakawa, H., Kajimura, Y. and Kojima, H., "Thrust Evaluation of a Magnetic sail by Flux-Tube Model," Journal of Propulsion and Power, Vol. 28, No. 3, 2012, pp. 642-651.

[18] Saha, S., Singh, N., Craven, P., Gallagher, D., and Jones, J., "Development of 3D Hybrid Code and Its Application to M2P2," Space Technology and Applications International Forum-STAIF 2002, Albuquerque, NM, 2001, pp. 441-446.

[19] Omidi, N. and Karimabadi, H., "Kinetic Simulation/Modeling of Plasma Sail," 39th AIAA/ASME/SAE/ASEE Joint Propulsion Conference and Exhibit, Huntsville, Alabama, Paper 2003-5226, Jul, 2003.

[20] Funaki, I., Kajimura, Y., Ashida, Y., Nishida, H., Oshio, Y., Shinohara, I. and Yamakawa, H., "The Use of Dipole Plasma Equilibrium for Magnetic Sail Spacecraft", Transactions of Fusion Science and Technology, 2013 in press.

[21] Nagasaki, Y., Nakamura, T., Funaki, I., Ashida, Y. and Yamakawa, H., "Conceptual Design of YBCO Coil with Large Magnetic Moment for Magnetic Sail Spacecraft,” IEEE Trans. Appl. Supercond., accepted.

[22] Nagasaki, Y., Nakamura, T., Koyama, T., Funaki, I., Ashida, Y., Kojima, H. and Yamakawa, H., “Characteristic comparison between Bi-2223/Ag and YBCO system superconducting magnets for magnetic sail spacecraft," Asian Conference on Applied Superconductivity and Cryogenics 2011, New Delhi, India, P-122, Nov, 2011. 